\title{
Homo Oeconomicus versus Homo Reciprocans \\ Ansätze für ein neues wirtschaftspolitisches Leitbild?
}

\section{Working Paper}

Author(s):

Falk, Armin

Publication date:

2001

Permanent link:

https://doi.org/10.3929/ethz-a-004374014

Rights / license:

In Copyright - Non-Commercial Use Permitted

Originally published in:

Working paper / Institute for Empirical Research in Economics 79 


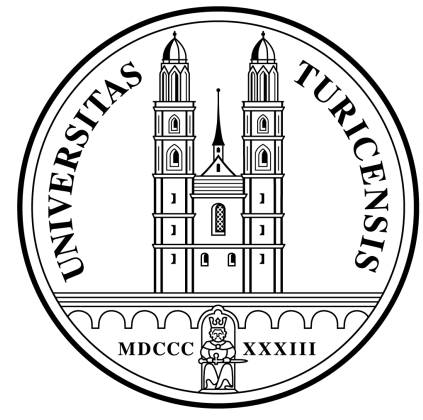

Institute for Empirical Research in Economics

University of Zurich

Working Paper Series

ISSN 1424-0459

Working Paper No. 79

Homo Oeconomicus Versus Homo Reciprocans:

Ansätze für ein Neues Wirtschaftspolitisches

Leitbild?

Armin Falk

July 2001 


\title{
HOMO OECONOMICUS VERSUS HOMO RECIPROCANS:
}

\author{
ANSÄTZE FÜR EIN NEUES WIRTSCHAFTSPOLITISCHES
}

\section{LEITBILD?}

\author{
Armin Falk \\ Universität Zürich, CESifo, CEPR
}

Juli 2001

Institut für Empirische Wirtschaftsforschung

Universität Zürich

Blümlisalpstr. 10

CH-8006 Zürich

Email: falk@iew.unizh.ch

\section{Zusammenfassung}

Politik und öffentliches Bewusstsein werden zunehmend durch ökonomische Theorien und Handlungsvorschläge mitbestimmt. Kaum ein anderes erkenntnis- und handlungsleitendes Modell hat daher einen vergleichbaren Einfluss wie das Konzept des Homo Oeconomicus. Durch die Entwicklung experimenteller Methoden ist es möglich, die Annahmen dieses Konzepts unter kontrollierten Laborbedingungen mit dem tatsächlichen Handeln von Individuen zu vergleichen. In der vorliegenden Arbeit werden zunächst verschiedene Experimentalstudien diskutiert, die eindeutig belegen, daß der Homo Oeconomicus weitaus weniger universell ist, als gemeinhin angenommen. Die Mehrheit der Experimentalteilnehmer verhält sich reziprok, d.h. sie belohnt faires Verhalten und bestraft unfaires Verhalten, selbst wenn dies mit Kosten verbunden ist. Der Nachweis reziproken Verhaltens hat weitreichende Konsequenzen für die ökonomische Politikberatung. In dieser Arbeit werden u.a. die folgenden Implikationen diskutiert: (i) Steuermoral ist eine Form bedingter Kooperation und kann durch politische Partizipation sowie ein ,faires“ Steuersystem verstärkt werden. (ii) Sozialstaatliche Arrangements können sich einer breiten Akzeptanz versichern, wenn fundamentale Fairnessgrundsätze nicht verletzt werden und Leistungen an den Willen zur Selbsthilfe geknüpft werden. (iii) Reziprozität ermöglicht eine gewisse Vertragsdurchsetzung bei unvollständigen Verträgen. Explizite Anreizverträge können diese freiwilligen Formen der Durchsetzung u.U. unterminieren. (iv) Aus der Existenz des Homo Reciprocans folgt, daß Gesellschaften über informelle Mechanismen zur Durchsetzung von Normen und Regeln verfügen. Die politische Aufwertung kleiner politischer Einheiten (Subsidiarität) könnte zu einer verstärkten Nutzung dieser Mechanismen führen. (v) Reziprozität kann erklären, warum es auf Arbeitsmärkten, unabhängig von staatlichen Eingriffen, zu Lohnstarrheiten, nicht-kompensierenden Lohndifferentialen und unfreiwilliger Arbeitslosigkeit kommen kann. 


\section{Einleitung}

Über kaum ein anderes Konzept herrscht in den modernen Wirtschaftswissenschaften so weitgehende Einigkeit und Akzeptanz wie über das Konzept des Homo Oeconomicus. Es ist deshalb nicht verwunderlich, daß die mit diesem Konzept verbundenen Annahmen eines rationalen und eigennutzorientierten Individuums die Grundlage nahezu sämtlicher in den Wirtschaftswissenschaften erstellten und verwendeten Modelle bilden. Dies gilt unterschiedslos für alle Bereiche der Wirtschaftswissenschaften und über diese hinaus, z.B. in der Soziologie oder der Rechtswissenschaft. Wirtschaftswissenschaftliche Modelle bilden die Grundlage nicht nur für unser Verständnis sozialer Realität, sondern ebenfalls für die hieraus abgeleitete Politikberatung. Folglich hat die Annahme eines allgegenwärtigen Homo Oeconomicus nicht nur eine erkenntnisleitende Funktion, sondern bestimmt - vermittels Beratung und Sozialisation durch ökonomische Ausbildung - auch das Handeln politischer und unternehmerischer Entscheidungsträger.

$\mathrm{Ob}$ sich Menschen tatsächlich entsprechend der Homo Oeconomicus Annahmen verhalten, ist indes eine empirische Frage. Durch die Entwicklung moderner wirtschaftswissenschaftlicher Experimental-Techniken hat diese Frage eine entscheidende Wende erhalten. Labor-Experimente erlauben es, individuelles Verhalten in einer kontrollierten Umgebung zu studieren und mit theoretischen Prognosen zu vergleichen. Da die Bezahlung der Teilnehmer von ihren Entscheidungen in den Experimenten abhängt, ist es insbesondere möglich, in ökonomisch relevanten Entscheidungssituationen die Vorhersagen des Homo Oeconomicus-Konzepts direkt zu testen. ${ }^{1}$

Da die Methode der experimentellen Wirtschaftsforschung noch relativ neu ist, wird in Abschnitt 2 dieser Arbeit zunächst kurz auf die experimentelle Methode eingegangen. Abschnitt 3 diskutiert fünf Experimentalstudien. Es zeigt sich, daß die Mehrheit der Experiment-Teilnehmer sich nicht gemäss den Annahmen des Homo Oeconomicus verhält. Stattdessen zeigen die Experimental-Teilnehmer ein auf Reziprozität basierendes Verhaltensmuster. Unter Reziprozität wird ein Verhalten subsumiert, bei dem freundliches oder kooperatives Verhalten belohnt und unkooperatives oder unfreundliches Verhalten bestraft wird. Entscheidend hierbei ist, daß der „Homo Reciprocans“ auch dann belohnt oder bestraft, wenn dies für ihn mit materiellen Kosten verbunden ist. ${ }^{2}$

Die Experimente illustrieren Verhaltens-Prinzipien, die in Abschnitt 4 auf verschiedene politikrelevante Bereiche übertragen werden. Im Mittelpunkt stehen hierbei die folgenden Fragen: Wie lässt sich die Steuermoral, verstanden als eine Form bedingter Kooperation, verbessern? Wie gelingt es, die Akzeptanz gegenüber sozialpolitischen Maßnahmen zu erhöhen und welche Grundsätze ergeben sich aus der Existenz von Reziprozität für eine als gerecht wahrgenommene Sozialpolitik? Welche Alternativen zur ökonomischen Theorie der Kriminalität lassen sich mit Hilfe des Homo Reciprocans ableiten und welche Bedeutung erhalten neue Politikinstrumente (wie z.B.

\footnotetext{
1 Für eine prägnante und ausführliche Diskussion des Homo Oeconomicus Konzepts und seiner Implikationen s. Kirchgässner (1991).

${ }^{2}$ Eine präzise Definition von Reziprozität in einem spieltheoretischen Modell, sowie eine Anwendung dieses Modells auf empirische Regularitäten findet sich in Falk und Fischbacher (1999). An dieser Stelle soll nur darauf hingewiesen werden, daß Reziprozität kein (unbedingter) Altruismus ist. Zudem ist anzumerken, daß Reziprozität auch in nicht-wiederholten Spielen auftritt und deshalb etwas anderes ist, als „kooperative“ Gleichgewichtsstrategien in wiederholten Spielen, wie sie etwa durch die sog. FolkTheoreme oder das Konzept des „reciprocal altruism“ (Trivers 1971) beschrieben werden.
} 
Belief Management)? Was bedeutet die Existenz reziproker Fairness für die Einführung expliziter Leistungsanreize? Welche Konsequenzen hat der Nachweis informeller Sanktionsmechanismen für die Subsidiaritätsdebatte z.B. in Form einer Aufwertung kleinerer politischer Einheiten? Welche Bedeutung hat der Homo Reciprocans für die Funktionsweise von Märkten, insbesondere bei unvollständigen Vertragsmärkten wie dem Arbeitsmarkt? Abschnitt 5 fasst die wesentlichen Ergebnisse zusammen und diskutiert mögliche Missverständnisse, die mit dem Konzept des Homo Reciprocans verbunden sein können.

\section{Die Methode der experimentellen Wirtschaftsforschung}

$\mathrm{Zu}$ einem wirtschaftswissenschaftlichen Labor-Experiment wird eine Gruppe von Teilnehmern eingeladen. ${ }^{3}$ Im Experimental-Labor erhalten die Teilnehmer Instruktionen, die den genauen Ablauf des Experiments, die Informationsbedingungen sowie die Entscheidungs- und Auszahlungsregeln beschreiben. Kontrollfragen, die vor dem eigentlichen Beginn des Experiments beantwortet werden müssen, stellen sicher, daß alle Teilnehmer die Regeln des Experiments verstanden haben. Nachdem alle Teilnehmer sich mit den Regeln vertraut gemacht haben, treffen sie ihre Entscheidungen. Ein Experiment ist im Grunde nichts anderes als ein Spiel: Der Experimentator legt die Anzahl der Spieler fest, deren Strategienraum sowie die Auszahlungsfunktionen. Von zentraler Bedeutung ist die Tatsache, daß die Teilnehmer entsprechend der festgelegten Auszahlungsfunktionen tatsächlich Geld erhalten, d.h. alle Entscheidungen der Teilnehmer haben unmittelbar monetäre Konsequenzen. ${ }^{4}$ Letzteres unterscheidet wirtschaftswissenschaftliche Experimente von Umfragestudien oder sozialpsychologischen Experimenten. Während es z.B. „billig“ ist, in einer Umfrage zu behaupten, man sei ein kooperativer Mensch, fordert die gleiche Willensäußerung im Experiment ein Verhalten, welches das entsprechende Individuum Geld kostet. Es geht in Experimenten nicht um Bekenntnisse, sondern um reales Verhalten mit realen Konsequenzen.

Die besondere Stärke experimenteller Techniken liegt in der Möglichkeit, Verhalten in einer kontrollierten Umgebung zu studieren. Keine andere empirische Methode erlaubt ein vergleichbares Maß an Kontrolle. So bestimmt der Experimentator beispielsweise, welche monetären Konsequenzen ein bestimmtes Verhalten im Experiment hervorruft. Er kann präzise festlegen, welche Informationen die Teilnehmer in einer bestimmten Entscheidungssituation haben, er kontrolliert Einflussgrößen wie Anonymität und Kommunikationsbedingungen und er bestimmt, ob ein Spiel wiederholt oder einmalig durchgeführt wird. Die weitgehende Kontrolle erlaubt die Implementierung der ceteris paribus Klausel. ${ }^{5}$ So ist es möglich, den Verhaltenseinfluss

\footnotetext{
${ }^{3}$ Zur Experimentellen Methodik vgl. z. B. Kagel und Roth (1995), Davis und Holt (1993), Friedman und Sunder (1994) oder Falk und Tyran (1997).

${ }^{4}$ Häufig werden experimentelle Ergebnisse mit dem Hinweis kritisiert, es ginge um verhältnismäßig wenig Geld. Hierzu ist zu sagen, daß die Geldbeträge für die betreffenden Personen so gering nicht sind (der Verdienst entspricht in etwa dem eines typischen Studenten-Jobs). Zudem ist die Höhe des Geldbetrages für eine theoretische Prognose irrelevant, solange Nichtsättigung im Geld angenommen wird (vgl. Smith (1976)). Vor allem aber ist darauf hinzuweisen, daß die Ergebnisse von Experimenten sich kaum unterscheiden, egal ob es um ,typische“ Beträge geht oder um Geldbeträge in Höhe mehrerer Monatsgehälter (vgl. z.B. Fehr und Tougareva (1995)).

${ }^{5}$ Die hervorragenden Kontrollmöglichkeiten innerhalb eines Experiments implizieren typischerweise ein „,artifizielles“ Entscheidungsumfeld, das die Frage der externen Validität von Experimenten aufwirft. Hier wird ein trade-off sichtbar. Je reichhaltiger und „realistischer“ das Entscheidungsumfeld, desto schwieriger ist eine exakte Kontrolle.
} 
einer bestimmten Variable exakt zu ermitteln. Die folgenden Beispiele werden dies verdeutlichen.

\section{Experimentelle Evidenz}

In diesem Abschnitt werden fünf Experimente vorgestellt. Die Experimente liefern jeweils den Nachweis eines allgemeinen Verhaltensprinzips. Alle diskutierten Prinzipien wurden in zahlreichen weiteren Experimenten bestätigt. Den ExperimentBeschreibungen wird das entsprechende Prinzip vorangestellt.

Wenn ich im folgenden vom Homo Oeconomicus spreche, ist damit ein rationales und eigennutzorientiertes Individuum gemeint. Es ist richtig, daß das Konzept des Homo Oeconomicus im Prinzip viel weiter gefasst ist und auch nicht-egoistische Präferenzen berücksichtigen kann. Ich halte jedoch eine Einschränkung und Präzisierung des Konzepts aus drei Gründen für sinnvoll. Erstens wird der Homo Oeconomicus in den Wirtschaftswissenschaften und auch im Bewusstsein der Öffentlichkeit regelmäßig als eigennütziges Individuum modelliert und verstanden. Aller anders lautenden Einführungsbemerkungen von Lehrbüchern zum Trotz, bildet die Eigennutzhypothese de facto den Kern fast sämtlicher Modelle und Theorien. Zweitens wurde und wird immer wieder zurecht kritisiert, daß willkürliche Änderungen der Präferenzen das Konzept inhaltsleer und gegen Kritik immun machen. Kirchgässner (1991) hält hierzu beispielsweise fest, daß die Theorie ohne Festlegungen beliebig wird und „letztlich jegliches Verhalten erklärt“ (S. 59). Drittens gilt insbesondere im Hinblick auf die empirische Überprüfung der Homo Oeconomicus Annahmen (im Labor), daß eine exakte Festlegung der Präferenzen unerlässlich ist.

\subsection{Reziprozität: Belohnen und Bestrafen}

Prinzip 1. Die Mehrheit der Individuen belohnt faires und bestraft unfaires Verhalten, selbst wenn dies mit Kosten verbunden ist.

Das Verhaltensprinzip 1 soll anhand des folgenden Experiments verdeutlicht werden, das auf einem zweistufigen Zwei-Personen-Spiel basiert (vgl. Falk, Fehr und Fischbacher 2000b). ${ }^{6}$ Beide Spieler im Experiment (A und B) erhalten zunächst eine Ausstattung von 12 Punkten. Auf der ersten Stufe entscheidet Spieler A, ob er dem Spieler B Punkte geben oder wegnehmen möchte, wobei er jeden Punktebetrag zwischen 0 und 6 geben oder nehmen kann. Falls A sich entscheidet, B $a$ Punkte zu geben, werden diese Punkte vom Experimentator verdreifacht, so daß sich das Einkommen von B um $3 a$ erhöht, während sich das Einkommen von A um $a$ verringert. Entscheidet sich A hingegen, B $a$ Punkte wegzunehmen, erhält A zusätzlich $a$ Punkte, während sich das Einkommen von B um $a$ verringert. Auf der zweiten Stufe kann Spieler B nun seinerseits das Verhalten von Spieler A belohnen oder bestrafen. Eine Belohnung in Höhe von $b$ Punkten transferiert $b$ Punkte von B zu A. Eine Bestrafung in Höhe von $b$ Punkten hingegen kostet $\mathrm{B} b$ Punkte, reduziert das Einkommen von A jedoch um $3 b$.

Gemäss den Annahmen des Homo Oeconomicus Modells gilt für dieses Experiment eine einfache Prognose: Ein eigennutzorientierter Spieler B wird weder belohnen noch bestrafen, da beides für ihn mit Kosten verbunden ist. Alle Spieler B wählen deshalb auf der zweiten Stufe $b=0$, unabhängig davon, wie sich die Spieler A entscheiden. Die Spieler A werden deshalb den Spielern B die maximal mögliche

\footnotetext{
${ }^{6}$ Dieses Experiment wurde zuerst von Abbink, Irlenbusch und Renner (2000) durchgeführt.
} 
Punktzahl wegnehmen (6 Punkte). Etwas zu geben wäre irrational, da es den eigenen Gewinn reduzieren würde. Weniger wegzunehmen wäre ebenfalls irrational, da Spieler A nichts zu befürchten hat. Die Homo Reciprocans Prognose kommt zu einem anderen Ergebnis. Auf der ersten Stufe etwas zu geben, muss als eine freundliche und zudem effizienzsteigernde Handlung angesehen werden. (Falls A den Höchstbetrag von 6 weitergibt, ist der gemeinsame Verdienst $12-6+12+6 * 3=36$, den man anschließend verteilen kann.) Etwas wegzunehmen gilt hingegen als eine unfaire Handlung ist. Ein Spieler B mit Präferenzen für Reziprozität wird folglich faires Verhalten von A belohnen und unfaires Verhalten bestrafen.

Das Hauptergebnis ist in Figur $1 \mathrm{zu}$ sehen: Die Linie mit den Vierecken (Intentional) gibt an, wie - in Abhängigkeit vom Verhalten der Spieler A - die Teilnehmer B den Gewinn der Spieler A auf der zweiten Stufe verändern. Es zeigt sich eindeutig ein reziprokes Verhaltensmuster. Die Spieler B belohnen freundliche und bestrafen unfreundliche Handlungen. Wenn z.B. ein Spieler A 6 Punkte von B wegnimmt, wird er von B so bestraft, daß sein Gewinn sich auf der zweiten Stufe um 9 Punkte reduziert. Gibt ein Spieler A hingegen z.B. 1 Punkt, erhält er hierfür etwa 2 Punkte zurück. Die Daten verwerfen somit eindeutig die auf den Annahmen des Homo Oeconomicus Modells beruhende Prognose $b=0$.

\subsection{Reziprozität und die Rolle von Intentionen und Verfahren}

Prinzip 2. Faires und unfaires Verhalten wird belohnt bzw. bestraft. Eine Handlung bzw. ein Ergebnis wird jedoch nur dann als fair bzw. unfair eingeschätzt, wenn die handelnde Person hierfür tatsächlich verantwortlich ist, d.h. wenn die betreffende Person sich auch anders hätte entscheiden können. Dies bedeutet, daß nicht nur die Konsequenzen von Handlungen und Ergebnissen für eine FairnessBeurteilung relevant sind, sondern auch Intentionen bzw. Verfahren.

Neben der Frage, ob sich die Teilnehmer im Experiment reziprok verhalten, wurde in dem in 3.1 diskutierten Experiment auch untersucht, ob ein Spieler B auch dann belohnt und bestraft, wenn Spieler A für seine Handlung nicht verantwortlich gemacht werden kann. Sind es allein die Konsequenzen von Spieler As Verhalten, die als fair oder unfair wahrgenommen werden, oder spielen seine Intentionen ebenfalls eine Rolle? Um diese Frage zu beantworten, wurden in Falk, Fehr und Fischbacher (2000b) die Ergebnisse des oben beschriebenen Experiments mit denen eines zweiten Experiments verglichen, das bis auf einen einzigen Unterschied identisch mit dem vorigen Experiment war: im zweiten Experiment bestimmte ein Zufallsmechanismus die Entscheidung von A. Wiederum saßen sich Spieler vom Typ A und Typ B gegenüber. Diesmal aber wurde die Entscheidung, ob und wie viel ein Spieler A einem Spieler B gibt oder wegnimmt ausgewürfelt. Die Spieler B hatten wiederum die Möglichkeit, A zu belohnen oder zu bestrafen.

Das Hauptergebnis dieses zweiten Experiments ist ebenfalls in Figur 1 dargestellt (s. die Linie mit den Punkten (Nicht Intentional)). Der Unterschied zum ,intentionalen Experiment" ist offensichtlich: Während die Spieler B im ersten Experiment belohnen und bestrafen, verhalten sie sich im zweiten Experiment nicht mehr reziprok. Wenn z.B. ausgewürfelt wurde, daß Spieler A Spieler B 6 Punkte wegnehmen muss, dann wurde das Einkommen von A durch die Spieler B im Median nicht verändert. Das heißt, dass $\mathrm{B}$ in den beiden Experimenten die exakt gleichen Konsequenzen des Verhaltens von A sehr unterschiedlich wahrnimmt, nämlich abhängig davon, ob A verantwortlich für 
seine Handlungen ist oder nicht. Verhält sich A ,aus freien Stücken“ unfair, wird er bestraft; kann er hingegen ,nichts dafür“, dann bleibt die Bestrafung aus.

Figur 1: Belohnungs- und Bestrafungsverhalten der Spieler $B$ in Abhängigkeit des Verhaltens der Spieler A (Medianwerte)

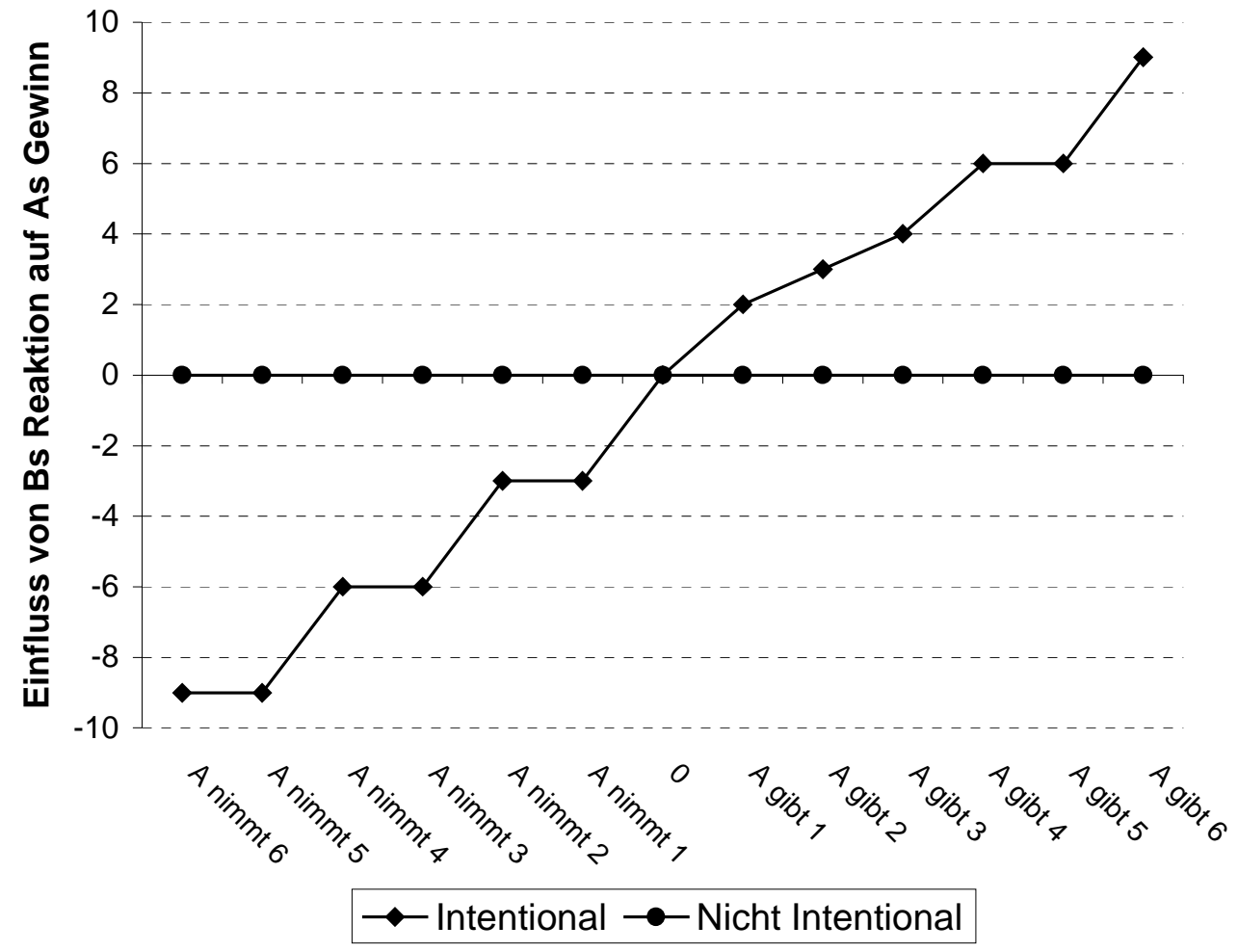

Diese Aussage hat für die Ökonomik eine weitreichende Bedeutung, weil Nutzenfunktionen Abbildungen aus dem Ergebnisraum sind, „Nutzen“ also konsequentialistisch definiert wird. ${ }^{7}$ Außerdem stellt die Tatsache, daß viele Menschen nicht nur die Ergebnisse von Handlungen und Entscheidungen betrachten, sondern bei ihren Überlegungen auch die Absicht berücksichtigeneine rein konsequentialistische Betrachtungsweise von Fairness in Frage. ${ }^{8}$ In engem Zusammenhang hiermit steht die Tatsache, daß die gleichen Ergebnisse unterschiedlich bewertet werden, abhängig davon, ob das zugrundeliegende Verfahren als fair wahrgenommen wird oder nicht (vgl. Lind und Tyler 1988). Diese sogenannte prozedurale Gerechtigkeit ist von politischer Bedeutung, da viele Menschen sehr viel eher bereit sind, auch unvorteilhafte Konsequenzen $\mathrm{zu}$ akzeptieren, wenn das zugrundeliegende Verfahren als fair und gerecht angesehen wird. Es ist $\mathrm{zu}$ erwarten, daß ein erhöhtes $\mathrm{Maß}$ an prozeduraler Gerechtigkeit sich positiv auf civic virtues wie etwa Steuermoral oder Freiwilligenarbeit auswirkt (Frey 1997).

\footnotetext{
${ }^{7}$ Ein theoretisches Modell, daß neben den Handlungsfolgen auch die Intentionen von Handlungen modelliert ist Falk und Fischbacher (1999). Die Bedeutung von Intentionen wurde auch in Falk, Fehr und Fischbacher (1999) aufgezeigt.

8 Eine ähnliche Differenzierung findet auch in der Rechtsprechung statt, bei der die gleichen Handlungsfolgen sehr unterschiedlich beurteilt werden, z.B. abhängig davon, ob die kriminelle Handlung aus Vorsatz oder Fahrlässigkeit erfolgte.
} 


\subsection{Reziprozität in Gruppen: Bedingte Kooperation und Multiple Gleichgewichte Prinzip 3: In sozialen Dilemma-Situationen zeigt die Mehrheit der Individuen ein bedingt kooperatives Verhalten: „Kooperieren die anderen, kooperiere ich auch; defektieren die anderen, defektiere ich auch." Hieraus ergibt sich u.U. die Existenz multipler Gleichgewichte, sogar in Situationen, in denen gemäss Standardtheorie nur ein einziges Gleichgewicht existiert.}

Die Bedeutung von Reziprozität ist nicht auf bilaterale Situationen beschränkt, sondern spielt auch in multilateralen Kontexten eine zentrale Rolle. Prototypisches Beispiel hierfür sind soziale Dilemma-Situationen, in denen es für das einzelne Individuum eine dominante Strategie ist, zu defektieren, während es für die Gruppe insgesamt am besten wäre, wenn alle kooperierten. Das folgende Experiment analysiert die Frage, welches Verhaltensmuster in einer solchen Situation von reziprok motivierten Individuen zu erwarten ist (vgl. Fischbacher, Gächter und Fehr 2001).

Die Teilnehmer werden in Vierergruppen eingeteilt. Jeder Teilnehmer erhält eine Ausstattung von 20 Punkten und die Möglichkeit zwischen 0 und 20 Punkte in ein öffentliches Gut zu investieren. Die Auszahlungsregel für ein Individuum ist: 20 Beitrag zum öffentlichen Gut + 0,4(Summe aller Beiträge zum öffentlichen Gut). Aus der Auszahlungsregel folgt unmittelbar, daß es für jeden Teilnehmer der Gruppe individuell besser ist, nichts zum öffentlichen Gut beizutragen, da jeder Punkt, der beigetragen wird 1 Punkt kostet, aber nur 0,4 Punkte einbringt. Da jedoch jeder der vier Gruppenteilnehmer von einem investierten Punkt profitiert, ist der Gruppengewinn dann am höchsten, wenn alle voll beitragen. In diesem Fall ist der Gewinn pro Spieler 20 $20+0,4(4 * 20)=32$, während er nur $20-0+0,4(0)=20$ beträgt, wenn alle defektieren. Es handelt sich mithin um ein typisches soziales Dilemma: Die individuell dominante Strategie nichts beizutragen, führt kollektiv zu einem ineffizienten Ergebnis.

Die Homo Oeconomicus Prognose in diesem Setup ist trivial. Jeder Spieler trägt 0 Punkte bei, unabhängig vom Verhalten der anderen Gruppenmitglieder. Das einzige Nash-Gleichgewicht ist folglich durch vollständiges Defektieren gekennzeichnet. Ein reziprok motiviertes Individuum hingegen stellt folgende Überlegung an: Der Beitrag eines anderen zum öffentlichen Gut ist ein freundlicher und kooperativer Akt, der durch einen eigenen Beitrag belohnt wird, d.h. ein reziprokes Individuum trägt dann bei, wenn auch die anderen beitragen. Das entsprechende Verhaltensmuster ist eine Strategie der bedingten Kooperation. Anders als ein unbedingter Altruist trägt ein reziprokes Individuum nur dann bei, wenn auch die anderen ihren Beitrag leisten. Wenn die anderen nicht beitragen, trägt auch ein reziprokes Individuum nichts bei, da es nicht der „Dumme“ sein möchte (Ausbeutungsaversion).

Im Experiment können die Teilnehmer ihren Beitrag zum öffentlichen Gut davon abhängig machen, wie viel die anderen Gruppenmitglieder zum öffentlichen Gut beitragen. Das Ergebnis ist in Figur 2 festgehalten. Obwohl gemäss den Annahmen des Homo Oeconomicus Modells niemand etwas beitragen sollte, erkennt man deutlich ein bedingtes Kooperationsverhalten. Im Durchschnitt trägt ein Teilnehmer um so mehr zum öffentlichen Gut bei, je mehr die anderen beitragen.

9 Dieses Verhalten wurde auch in einem ,Diebstahl-Experiment“ beobachtet, in dem gezeigt werden konnte, daß die Experimentteilnehmer um so mehr von anderen stahlen (defektierten), je mehr ihnen von anderen gestohlen wurde. Auch hier ist bedingte Kooperation das vorherrschende Muster (vgl. Falk und Fischbacher 2001). 
Figur 2: Bedingt kooperatives Verhalten: Durchschnittliche Beiträge in Abhängigkeit vom Verhalten der anderen Gruppenmitglieder

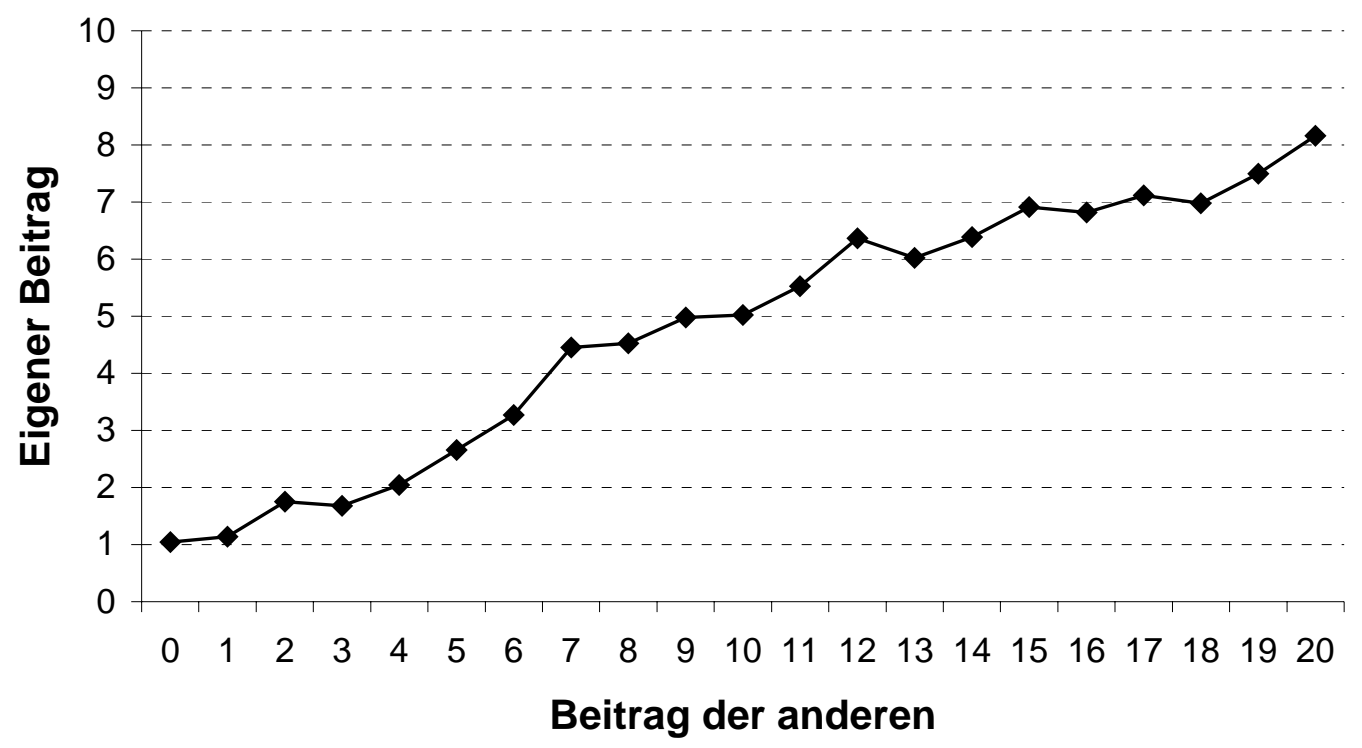

Aus bedingt kooperativem Verhalten folgt unmittelbar die mögliche Existenz multipler Gleichgewichte. Wenn genügend bedingt kooperative Typen in einer Gruppe zusammen sind, kann es sein, daß entweder wenig (oder nichts) beigetragen wird - weil die Erwartung vorherrscht, daß insgesamt wenig kooperiert wird - oder daß viel beigetragen wird, da insgesamt ein hohes Kooperationsniveau erwartet wird. Formal ausgedrückt ist ein Dilemma-Spiel, z.B. das Gefangenendilemma, das von zwei reziproken Spielern gespielt wird, tatsächlich ein Koordinationsspiel: Es gibt das „gute“ Gleichgewicht, bei dem beide kooperieren und das „schlechte“ Gleichgewicht, bei dem beide defektieren. Das bedeutet, dass es multiple Gleichgewichte in Spielen gibt, in denen es gemäss den Annahmen des Homo Oeconomicus Modells nur ein einziges (ineffizientes) Gleichgewicht geben kann. Da es bei multiplen Gleichgewichten entscheidend auf die Erwartungen über das Verhalten der anderen ankommt, spielt die Beeinflussung dieser Erwartungen durch Belief Management eine zentrale Rolle (s. Abschnitt 4.1 und 4.4.).

\subsection{Reziprozität auf Märkten: Vollständige vs. Unvollständige Vertragsmärkte} Prinzip 4: Reziprozität spielt auch für das aggregierte Ergebnis auf Märkten eine Rolle. Entscheidend ist jedoch, ob es einen Spielraum für reziproke Reaktionen gibt: Während Reziprozität auf unvollständigen Vertragsmärkten das Marktergebnis beeinflussen kann, gilt dies auf vollständigen Vertragsmärkten kaum.

Dieser Abschnitt behandelt den Einfluss von Reziprozität in Märkten. Ein besonderes Augenmerk wird dabei auf den Unterschied zwischen einem vertraglich vollständig geregelten Markt und einem vertraglich unvollständig geregelten Markt gelegt (vgl. Fehr und Falk 1999). 
Zunächst verhandeln 11 Verkäufer und 7 Käufer auf einem Wettbewerbsmarkt. ${ }^{10}$ Akzeptiert ein Verkäufer das Preisangebot eines Käufers oder akzeptiert ein Käufer das Angebot eines Verkäufers, ist zwischen beiden automatisch ein Vertrag zum vereinbarten Preis geschlossen. Da jeder Käufer mit nur jeweils einem Verkäufer einen Vertrag abschließen kann, gibt es auf dem Markt ein Überschussangebot von 4 Verkäufern.

Im Experiment mit unvollständigen Verträgen müssen alle Verkäufer, die einen Vertrag abgeschlossen haben, eine Qualität wählen. Während also der Preis, den ein Käufer „seinem“ Verkäufer zahlt, auf der ersten Stufe festgelegt wird, ist der Verkäufer in der Wahl der Qualität völlig frei. In diesem Sinn ist der Vertrag unvollständig. ${ }^{11}$ Die Wahl der Qualität $q$ wird durch eine Zahl zwischen 0,1 und 1 repräsentiert, wobei die Kosten der Qualität $c(q)$ für den Verkäufer mit der Höhe der gewählten Qualität steigen. Für $q=0,1$ belaufen sich die Kosten auf 0. Der Gewinn, den ein Käufer macht, wenn er das Gut kauft, beträgt (120 - Preis)q. Ein Verkäufer erhält den Preis abzüglich der Kosten der gewählten Qualität, d.h. Preis - $c(q)$. Der Reservationsnutzen eines Verkäufers beträgt 20. Beim vollständigen Vertragsmarkt wird die Qualität exogen festgelegt, d.h. die Verträge sind vollständig, und die Gewinne belaufen sich auf 120 Preis für die Käufer und Preis - 20 für die Verkäufer.

Das Homo Oeconomicus Modell prognostiziert sowohl für den vollständigen wie auch den unvollständigen Vertragsmarkt die selben Preise: Für Verkäufer im unvollständigen Vertragsmarkt gibt es keinen Grund, eine höhere als die minimale Qualität zu wählen, da höhere Qualitäten mit zunehmenden Kosten verbunden sind. Folglich werden alle Verkäufer auf der zweiten Stufe unabhängig vom gezahlten Preis $q$ $=0,1$ wählen. Rationale Käufer antizipieren die Wahl von $q=0,1$ und versuchen, den tiefstmöglichen Preis durchzusetzen, der gerade noch akzeptiert wird. Da der Reservationsnutzen der Verkäufer auf beiden Märkten 20 beträgt und es ein Überschussangebot an Verkäufern gibt, können Käufer Löhne in Höhe von 20 durchsetzen. Dieser Zusammenhang wird in Figur 3 verdeutlicht, welche die im Experiment implementierten Angebots- und Nachfragekurven zeigt. Zusammengefasst lautet die Prognose folglich für beide Vertragsmärkte, daß sieben Verkäufer zu einem Preis von 20 verkaufen und daß im unvollständigen Vertragsmarkt alle Verkäufer die minimale Qualität $q=0,1$ wählen.

\footnotetext{
${ }^{10}$ Der Markt wurde in einer sogenannten Doppelauktion organisiert. Das bedeutet, daß alle Verkäufer und Käufer gleichzeitig Angebote unterbreiten und jedes Angebot der Gegenseite akzeptieren konnten. Die Doppelauktion generiert einen höheren Wettbewerbsdruck als jede andere Institution der experimentellen Wirtschaftsforschung (vgl. z.B. Friedman und Rust 1993).

11 Die Annahme, daß viele Verträge (zum Beispiel Arbeitsverträge) unvollständig sind ist weithin anerkannt (vgl. z. B. Milgrom und Roberts 1992).
} 
Figur 3: Angebots- und Nachfragekurven für den vollständigen und den unvollständigen Vertragsmarkt

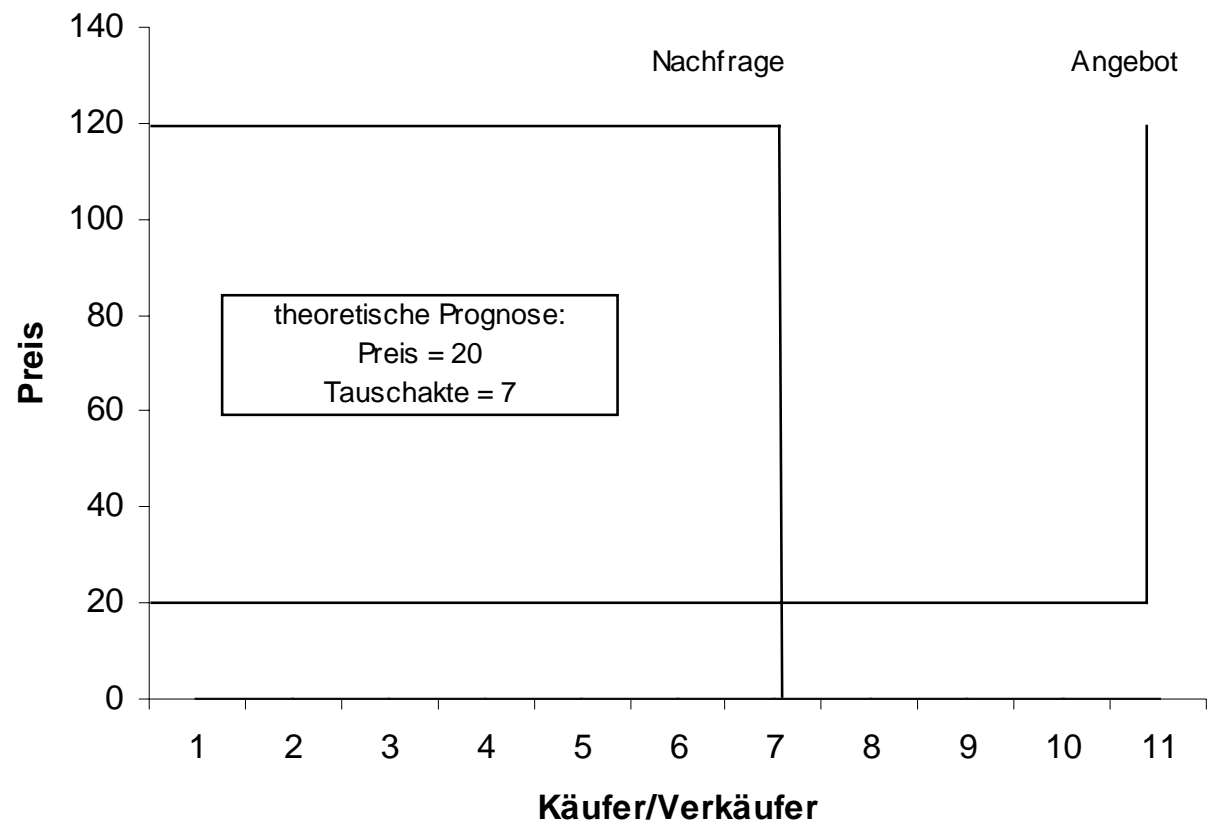

Das tatsächlich beobachtete Verhalten im unvollständigen Vertragsmarkt weicht deutlich vom prognostizierten Verhalten ab. Der durchschnittlich gezahlte Preis beträgt nicht 20, sondern 61, d.h. er ist drei mal höher als das Homo Oeconomicus Modell vorhersagt. Das ist ein bemerkenswertes Resultat. Trotz starkem Wettbewerb und einem deutlichen Überschussangebot seitens der Verkäufer konvergieren die Preise nicht zum prognostizierten Gleichgewichtswert. Dies ist um so überraschender, als sich die Verkäufer im Experiment tatsächlich stark gegenseitig unterboten haben und Käufer problemlos tiefere Preise hätten zahlen können. Auf die Frage, warum die Käufer freiwillig höhere Preise zahlen, gibt Figur 4 eine einfache Antwort. Sie zeigt die durchschnittlich gewählte Qualität in Abhängigkeit vom gezahlten Preis. Das reziproke Muster ist klar erkennbar. Je höher der gezahlte Preis ausfällt, desto höher ist im Durchschnitt die geleistete Qualität. Wenn die Verkäufer einen „fairen“ Preis erhalten, sind sie auch bereit, eine „faire“ Qualität zu liefern. Die positive Beziehung von Preis und Qualität lässt es für Käufer nicht lukrativ erscheinen, tiefe Preise durchzusetzen. Stattdessen versuchen sie, durch höhere Preise an die Reziprozität der Verkäufer zu appellieren. Tatsächlich ist die im Experiment beobachtete Korrelation zwischen dem von den Käufern gezahlten Preis und ihrem Profit positiv.

Wenn die positive Beziehung zwischen Preis und Qualität dafür verantwortlich ist, daß im unvollständigen Vertragsmarkt höhere Preise gezahlt werden, dann wäre zu erwarten, daß im Falle vollständiger Verträge die Preise tiefer sind, da es hier für die Käufer keinen Grund gibt, an die Reziprozität der Verkäufer zu appellieren. Das ist tatsächlich der Fall. Während die Käufer im unvollständigen Vertragsmarkt freiwillig „faire“ Preise zahlen, drücken sie die Preise im vollständigen Vertragsmarkt so tief wie sie können. Als Ergebnis dieser Politik konvergieren die durchschnittlichen Preise relativ gut zum prognostizierten Gleichgewicht.

Die allgemeine Einsicht aus dem Vergleich dieser beiden Experimente lässt sich wie folgt zusammenfassen: Bei unvollständigen Verträgen gibt es einen Spielraum für 
Reziprozität, weshalb das Marktergebnis sich deutlich von der Prognose des Homo Oeconomicus Modells unterscheidet. Bei vollständigen Verträgen hingegen fehlt dieser Spielraum, sodass es für die Käufer keinen Grund gibt, hohe Preise zu zahlen. Nur für auf vollständigen Vertragsmärkten wird daher das aggregierte Verhalten relativ gut durch die Prognose des Homo Oeconomicus Modells erfasst. ${ }^{12}$

\section{Figur 4: Beziehung zwischen Preis und Qualität}

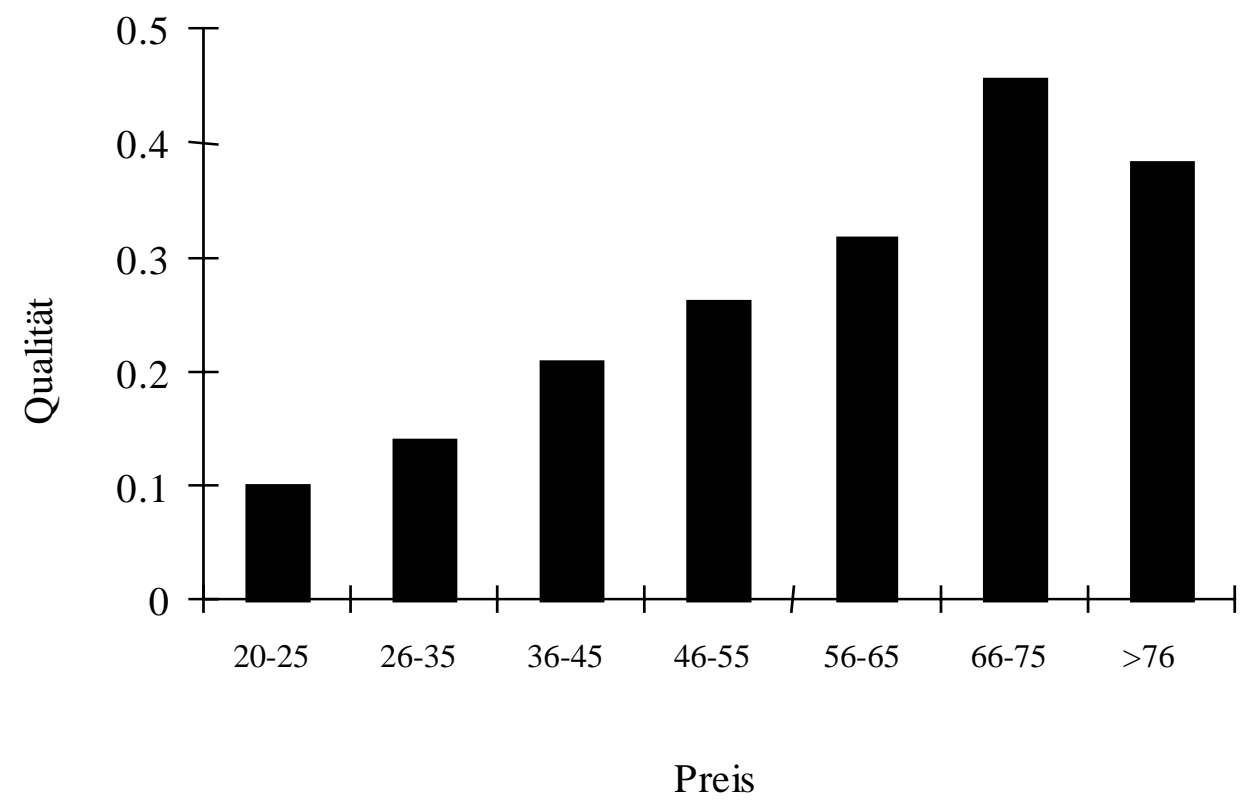

\subsection{Reziprozität und Eigennutz: Interaktion von Typen}

Prinzip 5: In Gruppen, in denen (wie es typischerweise der Fall ist) eigennützige und reziproke Individuen miteinander interagieren, hängt es vom institutionellen Umfeld ab, ob sich reziprokes oder eigennütziges Verhalten „durchsetzt": In sozialen Dilemma-Situationen ohne individuelle Bestrafungsmöglichkeiten wird sich häufig eigennütziges Verhalten durchsetzten. Existiert hingegen die Möglichkeit zu (informellen) Sanktionen, können Egoisten durch reziproke Individuen diszipliniert werden, sodass Kooperation erreicht wird.

In den bisher diskutierten Experimenten gilt, dass Reziprozität zwar das vorherrschende Verhaltensmuster ist, eine Minderheit aber ein mit den Annahmen des Homo Oeconomicus-Modells kompatibles Verhalten zeigt. Es stellt sich deshalb die Frage, ob in sozialen Dilemma-Situationen reziproke oder eigennutzorientierte Individuen das aggregierte Verhalten determinieren. Das folgende Experiment zeigt, dass dies vom institutionellen Umfeld abhängt.

In dem Experiment gibt es zwei verschiedene Bedingungen: Unter der „StrafBedingung" haben die Teilnehmer die Möglichkeit, andere Gruppenmitglieder zu bestrafen, während sie unter der Kontrollbedingung diese Möglichkeit nicht besitzen

\footnotetext{
12 Der Unterschied zwischen vollständigen und unvollständigen Vertragsmärkten wird durch formale Fairnesstheorien prognostiziert (vgl. Falk und Fischbacher 1999).
} 
(vgl. Fehr und Gächter 2000c). Die Parameter sind dieselben wie im Experiment in Abschnitt 3.3. Die Gruppen bestehen aus jeweils vier Teilnehmern; jeder Teilnehmer erhält eine Ausstattung von 20 Punkten und kann zwischen 0 und 20 Punkten in ein öffentliches Gut investieren, das allen zugute kommt. Die Auszahlungsfunktion für ein Individuum ist 20 - Beitrag zum öffentlichen Gut + 0,4(Summe aller Beiträge zum öfentlichen Gut). Unter beiden Bedingungen interagieren die vier Gruppenmitglieder über insgesamt 10 Perioden miteinander, wobei sie nach jeder Periode darüber informiert werden, wie sich die anderen Gruppenmitglieder verhalten haben. Der einzige Unterschied zwischen den Bedingungen ist der, daß sich in der Strafbedingung alle Teilnehmer gegenseitig bestrafen können, in dem sie sich gegenseitig Punkte abziehen. Diese ,informellen Sanktionen“ sind allerdings mit Kosten verbunden. Aus diesem Grund prognostiziert die Standardtheorie, dass erstens in beiden Bedingungen niemand etwas zum öffentlichen Gut beiträgt und dass zweitens unter der Strafbedingung niemand einen anderen Teilnehmer straft. ${ }^{13}$

Wie sich die Individuen tatsächlich in solch einem Experiment verhalten, ist Figur 5 ersichtlich. Die weißen Balken geben die relative Häufigkeit an, mit der unter der Kontrollbedingung ohne Strafmöglichkeit zum öffentlichen Gut beigetragen wird. Es zeigt sich, dass zwar einige Teilnehmer mehr beigetragen als mit purem Eigennutz vereinbar ist, die Mehrheit der Teilnehmer aber sehr wenig oder nichts beiträgt. So geben mehr als 50 Prozent aller Teilnehmer Beiträge von Null. Unter der „Strafbedingung“ hingegen ergibt sich ein radikal anderes Bild. Hier kooperiert die Mehrheit, und etwa 80 Prozent der Teilnehmer kooperieren sogar voll. Die Erklärung für diesen Unterschied liefert die Interaktion von eigennützigen und reziproken Individuen in Verbindung mit dem institutionellen Umfeld: Unter der Kontrollbedingung ohne Bestrafungsmöglichkeit tragen reziproke Teilnehmer am Anfang relativ viel bei, während die Egoisten nichts beitragen. Da reziproke Individuen eine starke Ausbeutungsaversion haben und nicht unbedingt, sondern nur bedingt kooperativ sind, reduzieren sie in den folgenden Perioden ebenfalls ihre Beiträge gemäss dem Motto: Wenn die anderen nicht beitragen, trage ich auch nicht bei niemand möchte der „Dumme“ sein. Hierdurch entsteht eine Abwärtsspirale, die ohne zusätzliche institutionelle Einrichtungen kaum zu durchbrechen ist. Anders unter der Strafbedingung: Hier haben die reziproken Teilnehmer die Möglichkeit, unfaires Verhalten zu bestrafen. Tatsächlich beobachtet man, daß Teilnehmer um so mehr bestraft werden, je weniger sie beitragen. Hierdurch kann die Mehrheit der reziproken Teilnehmer die egoistische Minderheit effektiv disziplinieren, so daß es auch im Eigeninteresse der Egoisten liegt, beizutragen. Als Ergebnis beobachtet man hohe Kooperationsraten unter der Strafbedingung und geringe in der Kontrollbedingung, obwohl die Zusammensetzung aus reziproken und egoistischen Teilnehmern exakt gleich ist. Zusammenfassend lässt sich somit sagen, daß Reziprozität als ein informeller Durchsetzungsmechanismus für kooperatives Verhalten fungieren kann. Wenn die Möglichkeiten zu informellen Sanktionen bestehen, verfügen Gruppen nicht nur über eine Bereitschaft zu freiwilliger Kooperation, sondern - und das ist besonders wichtig über die freiwillige Bereitschaft, Trittbrett-Fahren zu sanktionieren, selbst wenn die Ausübung der Sanktionen mit Kosten verbunden ist.

\footnotetext{
${ }^{13}$ Diese Prognose gilt mit Rückwärtsinduktion trotz der Wiederholung von 10 Perioden, da es sich um ein endlich oft wiederholtes Spiel handelt.
} 
Figur 5: Beiträge zum öffentlichen Gut unter der Straf- und der Kontrollbedingung

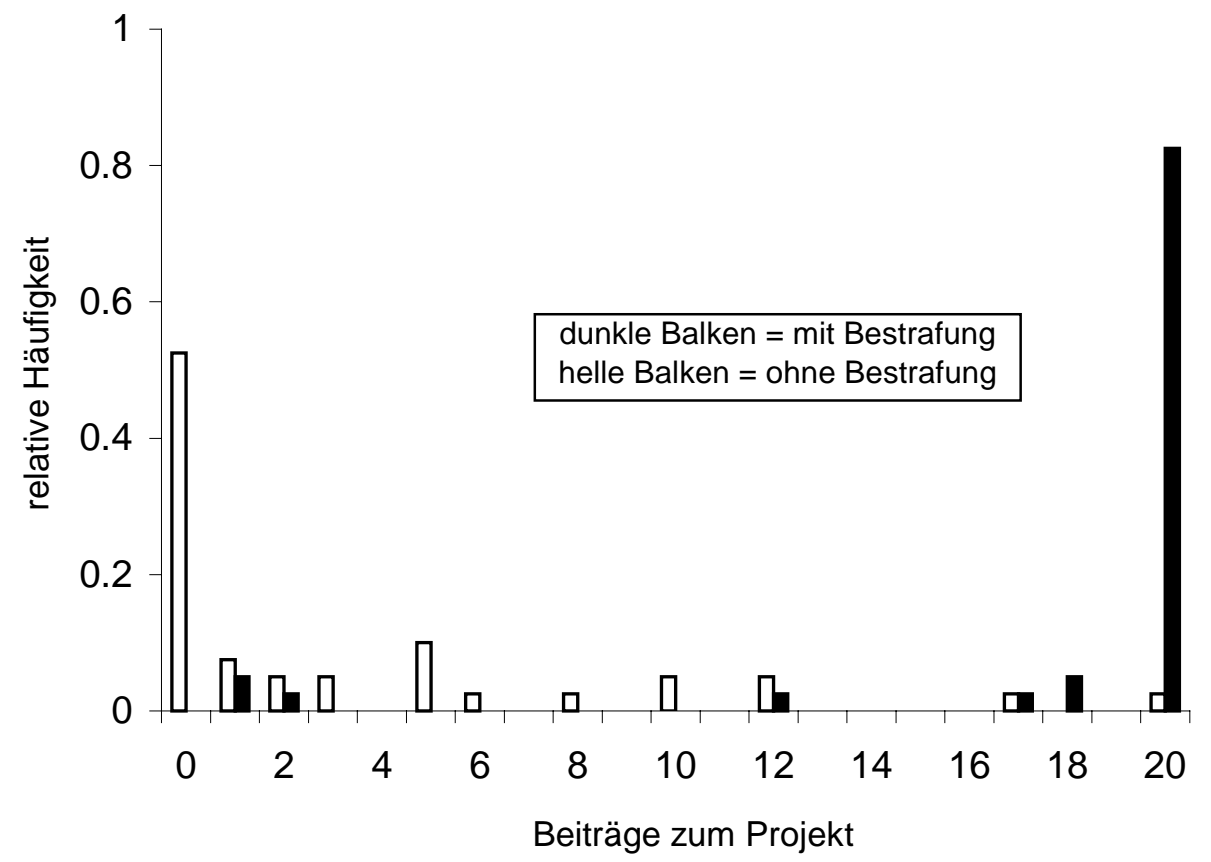

\subsection{Weitere Evidenz}

Die hier präsentierten Ergebnisse stellen nur einen Bruchteil der vorhandenen empirischen Evidenz dar. Die Existenz reziproken Verhaltens ist in Dutzenden von Experimenten, unter variierenden experimentellen Bedingungen und in verschiedenen Kulturen, gezeigt worden. ${ }^{14}$ Eines der bekanntesten Experimente ist das sogenannte Ultimatum Spiel (Güth et al. 1982), ein Verhandlungsspiel, bei dem eindrucksvoll gezeigt wurde, daß positive, aber als unfair wahrgenommene Angebote abgelehnt werden. Weitere Experimente, die die Bedeutung von Fairness zeigen sind z.B. Bolle und Kritikos (1998), Jacobsen und Sadrieh (1996) und Keser und Van Winden (2000). Sehr früh hat Selten (1978) auf die Relevanz von Fairness verwiesen. Bolle (1998) diskutiert die Rolle von Vertrauen. Neben der experimentellen Evidenz haben in letzter Zeit auch Fragebogenstudien, die mit Managern und Personalverantwortlichen von Firmen durchgeführt wurden, die ökonomische Relevanz von Fairness eindrücklich belegt (z.B. Bewely (1999)). Auch in Nachbarwissenschaften wie der Soziologie und Sozialpsychologie ist die Existenz und Bedeutung von Reziprozität gut dokumentiert. Stellvertretend für viele sei der Soziologe Gouldner (1960, S. 171) genannt, der die Überzeugung vertritt, daß Reziprozität als kulturelles Phänomen nicht weniger universell sei als das Inzestverbot. Reziproke Verhaltensmuster haben ihre tiefere Wurzel möglicherweise in der Evolutionsgeschichte des Menschen. ${ }^{15}$

\section{Implikationen für die Wirtschaftspolitik}

Die experimentell demonstrierten Verhaltensprinzipien werden im folgenden auf ihre Bedeutung für wirtschafts- und sozialpolitische Fragen hin reflektiert. Hierbei geht es nicht mehr um konkrete Experimentsituationen, sondern um die Analyse von Reziprozität als allgemeinem Verhaltensmuster. Alle abgeleiteten Implikationen sind als

\footnotetext{
${ }^{14}$ Für einen Überblick vgl. Fehr und Gächter (2000a).

${ }^{15}$ Siehe hierzu beispielsweise de Wal (1996), der bedingtes Kooperationsverhalten bei Primaten studiert.
} 
positive Analyse zu verstehen. Es geht nicht darum, zu formulieren was wünschenswert wäre, sondern darum, welche Konsequenzen sich aus der Co-Existenz von Homo Oeconomicus und Homo Reciprocans für verschiedene ökonomisch relevante Politikfelder ergeben können.

\subsection{Steuermoral als bedingte Kooperation}

Steuern bilden das finanzielle und politische Rückgrat moderner westlicher Gesellschaften. Aus diesem Grund stellt das Problem der Steuerhinterziehung ein zentrales wirtschaftspolitisches Problem dar. Schätzungen zufolge entgehen z.B. dem US-Haushalt aufgrund von Steuerhinterziehung Einnahmen in Höhe von ca. $130 \mathrm{Mrd}$. US\$ pro Jahr (vgl. Andreoni, Erard und Feinstein 1998). ${ }^{16}$ Für die Schweiz haben Untersuchungen ergeben, daß etwa 17,5 Prozent des Einkommens hinterzogen werden (Frey 1997). Neben diesen erheblichen fiskalpolitischen Problemen entstehen durch Steuerhinterziehung Steuerungerechtigkeiten. Zudem lassen sich SteuerInzidenzwirkungen nicht mehr verlässlich angeben.

Die traditionelle Analyse der Steuerhinterziehung richtet sich primär auf die Auswirkungen materieller Anreize, d.h. die Strafhöhe bei erwiesener Hinterziehung und die Aufdeckungswahrscheinlichkeit. Die in Abschnitt 3 präsentierte empirische Evidenz legt demgegenüber die Bedeutung freiwilliger Formen der Steuerzahlung nahe (Steuermoral). Offenbar gibt es eine bedingte Bereitschaft, zu öffentlichen Gütern beizutragen und das Verhalten von Trittbrett-Fahrern zu bestrafen, d.h. auch nichtmaterielle, informelle Faktoren spielen bei der Entscheidung, die Steuern zu zahlen bzw. zu hinterziehen, eine Rolle. ${ }^{17}$ Diese Feststellung deckt sich mit der Tatsache, daß weniger Steuern hinterzogen werden, als mit dem Homo Oeconomicus Modell vereinbar (vgl. Andreoni, Erard und Feinstein 1998).

Im folgenden soll Steuermoral als eine Form bedingt kooperativen Verhaltens verstanden werden. Es soll untersucht werden, wie Steuermoral durch die Wahl des Steuersystems und anderer staatlicher Institutionen sowie durch politisches Belief Management positiv beeinflusst werden kann.

Steuermoral als bedingte Kooperation impliziert, daß jemand um so eher bereit ist seine Steuern zu zahlen, je mehr er davon überzeugt ist, daß auch die anderen einen fairen Beitrag zum öffentlichen Gut zahlen, d.h. wenn auch die anderen einen fairen Steuerbeitrag leisten (s. Prinzip 3). Ob der Steuerbeitrag der anderen als „fair“ angesehen wird, hängt zunächst vom Steuertarif ab. Wenn die Steuertarife als fair betrachtet werden, d.h. wenn man glaubt, daß jeder nach seinen Möglichkeiten und seiner Leistungsfähigkeit herangezogen wird, erhöht das die Bereitschaft, seinen Teil beizutragen. Diese Überlegung hat weitreichende Konsequenzen für die Debatte um „optimale Steuern“. Die konventionelle ökonomische Analyse, bei der Fairness im allgemeinen keine Rolle spielt, argumentiert beispielsweise zugunsten einer Kopfsteuer, da sie die geringsten Verzerrungswirkungen aufweist. Die Effizienzwirkungen einer Kopfsteuer fallen indes völlig anders aus, wenn die Bedeutung von Steuermoral und

\footnotetext{
${ }^{16}$ Eng verwandt mit dem Problem der Steuerhinterziehung sind alle Aktivitäten, die mit dem Begriff der Schattenwirtschaft (,underground economic activities“) bezeichnet werden (vgl. hierzu Schneider und Enste 2000).

${ }^{17}$ Andreoni, Erard und Feinstein (1998) kommen in ihrem Überblicksartikel zu dem folgenden Ergebnis: „,...adding moral and social dynamics to models of tax compliance is as yet a largely underdeveloped area of research. There seems little dispute about whether these factors are important, but little is known or agreed upon about how best to include these effects in a theoretical or empirical analysis of tax compliance" (S. 852).
} 
Fairnessargumenten berïcksichtigt wird. Wird entsprechend einem Kopfsteuerregime besteuert, besteht die Möglichkeit, daß viele Steuerzahler nicht (mehr) den Eindruck haben, daß alle einen fairen Beitrag leisten. Insbesondere die sehr Wohlhabenden entrichten im Vergleich zur Mittelschicht nur noch einen sehr geringen Anteil ihres Einkommens. Es ist zu erwarten, daß bedingt kooperative Steuerzahler auf ein solchermaßen unfaires System reziprok, d.h. mit Steuervermeidung oder anderen für das Gemeinwohl nachteiligen Formen des Protests reagieren. Die Reaktionen auf die versuchte Einführung der poll tax durch die Thatcher-Regierung in England belegen diese Vermutung. Es kam zu starken Protesten, so daß die neue Steuer politisch nicht durchsetzbar war. In Anbetracht der Tatsache, daß Steuermoral für das gesamte Steueraufkommen in einem Land eine zentrale Rolle spielt, ist die Effizienzbeurteilung der Kopfsteuer viel schwieriger, als es aus Sicht der Standardperspektive suggeriert wird.

Diese Aussage gilt nicht nur für das extreme Beispiel der Kopfsteuer, sondern für alle Ausprägungen des Steuersystems. Alle Faktoren, die für die Fairness eines Steuersystems relevant sind, können sich auf die Steuermoral auswirken, sei es die absolute Höhe der Steuerbelastung, die Ausgestaltung der Steuertarife, der Eingangssteuersatz, der Höchststeuersatz oder konkrete Regeln wie das Ehegattensplitting. Aber auch die Ungleichbehandlung ganzer Berufsgruppen wie z.B. der Angestellten auf der einen und der Beamten auf der anderen Seite, können einen direkten Einfluss auf die Steuermoral haben. Wenn es richtig ist, daß die wahrgenommene Fairness des Steuersystems auf die Steuermoral einen Einfluss ausübt, ist die Erforschung, welche Eigenschaften eines Steuersystems als fair oder unfair wahrgenommen werden, von grosser politischer Bedeutung. Eine erste interessante Fragebogenstudie in dieser Richtung wurde von Seidl und Traub (1999) vorgelegt.

Aus der Annahme von Steuermoral als bedingter Kooperation folgt, wie oben dargelegt, die mögliche Existenz multipler Gleichgewichte (s. Prinzip 3). Anders als für den Homo Oeconomicus spielt es für den Homo Reciprocans eine wichtige Rolle, ob andere ihren Beitrag zu einem öffentlichen Gut leisten oder nicht. Wenn ein reziprokes Individuum den Eindruck hat, die anderen zahlten ihre Steuern, wird es sich ebenso verhalten (,gutes“ Erwartungsgleichgewicht). Herrscht hingegen der Eindruck vor, die anderen zahlten ihre Steuern nicht, wird ein reziproker Steuerzahler eine geringere Steuermoral aufweisen (,schlechtes“ Erwartungsgleichgewicht). Mit anderen Worten spielen für die Steuerzahler die Erwartungen über die Steuermoral der anderen Steuerzahler eine große Rolle. Diese Einschätzung bestätigen verschiedene empirische Untersuchungen, die der Sozialpsychologe Cialdini wie folgt zusammenfasst: ,admitted noncompliers are more likely to estimate high levels of noncompliance within the general public ... and there is a clear positive relationship between self-reported evasion and the tax evasion of friends and relatives... (Cialdini 1992, S. 215).

Aus der Existenz von ,guten“ und ,schlechten“ Erwartungsgleichgewichten ergibt sich für die Politik ein neuer Ansatzpunkt, nämlich durch entsprechendes BeliefManagement "gute“ Erwartungsgleichgewichte zu erzielen. Hiermit ist die positive Beeinflussung von Erwartungen über das Verhalten anderer gemeint, die ein entsprechendes Verhalten unterstützen kann. Es könnte z.B. in Anzeigen und Fernsehspots darauf hingewiesen werden, daß die meisten Bürger ihrer Steuerpflicht nachkommen und Steuerhinterziehung als unsozial, unfair und kriminell ablehnen. Hierbei könnte zusätzlich klar gemacht werden, was der Allgemeinheit aufgrund der Hinterziehung einzelner entgeht. Des weiteren könnte der Einschätzung entgegengewirkt werden, es gäbe ungestrafte Hinterziehung. Es ist nicht zu 
unterschätzen, welchen negativen Einfluss öffentlich bekannt gewordene und spektakuläre Fälle von Steuerhinterziehung auf die Steuermoral ausüben: Die durch sie provozierte Frage lautet nämlich, warum soll ich ehrlich sein, wenn selbst der bekannte Herr X seine Steuern hinterzieht. Die Erosion der Steuermoral kann durch derartige negative Erwartungsschocks in Gang gebracht werden. Hilfreich sind deshalb sowohl Strafen für Steuerhinterzieher sowie deren soziale Ächtung, z.B. durch öffentliche Kampagnen. Letzteres ist vor allem deshalb von Bedeutung, da es die normative Übereinstimmung, daß Steuerhinterziehung ein gemeinschaftsfeindlicher Akt ist, verstärkt und emotional verankert. ${ }^{18}$

Wie weiter oben ausgeführt, hängen die Fairnesswahrnehmungen nicht nur von Ergebnissen sondern auch von Verfahren ab (s. Prinzip 2). Der Homo Reciprocans ist viel eher bereit, auch unvorteilhafte Massenahmen zu akzeptieren (z.B. als „zu hoch“ wahrgenommene Steuern zu zahlen), wenn das Verfahren, das zu der entsprechenden Maßnahme geführt hat, als fair wahrgenommen wird. Mit anderen Worten lässt sich durch die geeignete Wahl politischer Institutionen und die dadurch erhöhte prozedurale Gerechtigkeit eine höhere Zustimmung zu politischen Entscheidungen erreichen, selbst wenn diese für einzelne ,unvorteilhaft" sind. An erster Stelle wären hier verbesserte Möglichkeiten der politischen Partizipation zu nennen, also direktdemokratische Elemente bei Abstimmungen sowie Bürgerbeteiligungen und Referenden. Es ist gezeigt worden, daß sich ein höheres $\mathrm{Ma} ß$ an politischer Mitbestimmung positiv auf die Steuermoral auswirkt (vgl. hierzu Frey 1997). ${ }^{19}$ Da die Partizipationschancen in repräsentativen Systemen weder auf Seiten der Steuereinnahmen noch auf Seiten der Steuerverwendung besonders ausgeprägt sind, ist die Identifikation mit dem Gemeinwesen relativ gering. Zudem wird das politische Verfahren als weniger fair erlebt im Vergleich zu jenen Verfahren, die über Möglichkeiten direkter Bürgerbeteiligung verfügen. Feld und Frey (2000) merken hierzu in ihrer Studie über das Verhältnis von Steuermoral und direkter Demokratie an: „Treating citizens respectfully can be expected to be more pronounced in polities with constitutional provisions for direct voter participation, like referenda and initiatives... . In such a system of direct democracy, taxpayers know that the public services they consume are worth the taxes they pay. Taxpayers therefore feel obliged to pay their taxes honestly" $\left(\right.$ S. 6) ${ }^{20}$. Es ist zu erwarten, daß verstärkte politische Partizipation auch zu einer höheren Steuermoral führt. Besonders im Hinblick auf das häufig beklagte „Demokratiedefizit“ im Rahmen des europäischen Einigungsprozesses, sollte unter diesem Gesichtspunkt die Chance der institutionellen Neugestaltung unter Einbezug direktdemokratischer Verfahren genutzt werden (vgl. Schneider 2000).

\subsection{Akzeptanz sozialpolitischer Maßnahmen}

Bevor es einen zentralen Sozialstaat gab, wurde soziale Sicherung in verhältnismäßig kleinen dezentralen Einheiten auf Gegenseitigkeit (Reziprozität) bereitgestellt (vgl. Sugden 1986). Diese beruhten auf der freiwilligen Einzahlung in einen gemeinsamen Fonds, aus dem man im Bedarfsfall (Krankheit, Arbeitsunfähigkeit, Arbeitslosigkeit

\footnotetext{
${ }^{18}$ Hiermit wird die soziale Bedeutung von Strafen angesprochen: „Punishment does more than impose disutility on the offender; it also expresses the community's moral condemnation“ (vgl. Kahan 1997, S. 383).

${ }^{19}$ Erste experimentelle Arbeiten, die auf den positiven Einfluss politischer Partizipation hinweisen, sind Alm et al. (1993) und Alm et al. (1999).

${ }^{20}$ Goette und Kucher (1998) haben für die Stadt Zürich festgestellt, daß eine positive Korrelation zwischen der Vertrauenswürdigkeit in die Stadtregierung und der Steuerehrlichkeit der Bürger existiert.
} 
usw.) Leistungen beziehen konnte. Ein solches System kann nur funktionieren, wenn gegenseitig kooperiert und Trittbrett-Fahren sanktioniert wird (vgl. Hechter 1987). Auch der zentralisierte und anonymisierte Sozialstaat westlicher Gesellschaften, ist auf ein Mindestmass an Kooperation angewiesen. Auch heute funktioniert das Konzept einer Solidargemeinschaft nur, wenn alle in der Gemeinschaft ihren Beitrag leisten, wenn es keinen exzessiven Missbrauch von Leistungen gibt und das sozialstaatliche Arrangement als „fair“ angesehen wird und sich folglich einer breiten Akzeptanz versichern kann. Die Akzeptanz des Sozialstaats ist keineswegs garantiert. In den USA beispielsweise stoßen zahlreiche soziale Programme auf die weitgehende Ablehnung der Bevölkerung (vgl. Bowles und Gintis 1998, Wax 2000). Auch in europäischen Staaten wird über die Bezahlbarkeit und Angemessenheit sozialstaatlicher Maßnahmen lebhaft diskutiert. Die hier vertretene These lautet, daß der Sozialstaat langfristig gesehen nur bestehen und sich einer breiten Akzeptanz versichern kann, wenn er als ein faires Umverteilungssystem wahrgenommen wird. Die Beantwortung der Frage, was „fair" in diesem Zusammenhang heißt, soll im Rückgriff auf die oben dargelegten Prinzipien erfolgen. Die Kernfrage hierbei lautet: Wann wird eine Inanspruchnahme des Sozialstaats als fair, wann als unfair bzw. gemeinschaftsfeindlich wahrgenommen?

Aus Prinzip 2 folgt, daß eine unkooperative oder eigennützige Handlung nicht als unfair betrachtet wird, wenn das entsprechende Individuum keine alternative Verhaltensmöglichkeit hatte, d.h. wenn die Handlungsfolgen außerhalb der eigenen Kontrolle lagen. Auf staatliche Leistungen und die Akzeptanz von wohlfahrtsstaatlichen Regelungen übertragen heißt das: Ein kollektives Umverteilungssystems wird als „fair“ empfunden, wenn gilt, daß nur derjenige Leistungen erhält, der nicht oder nur zu unzumutbaren Bedingungen für sich selbst sorgen kann. Anders formuliert berechtigt Bedürftigkeit alleine noch nicht zu Ansprüchen, sondern nur eine Bedürftigkeit, die nicht selbst verschuldet wurde, d.h. die durch Umstände hervorgerufen wurde, die jenseits der eigenen Kontrollierbarkeit liegen. Wenn beispielsweise Arbeitslosigkeit im eigenen Verantwortungsbereich liegt und trotzdem Ansprüche an das Kollektiv gestellt werden, so wird das als unfair betrachtet. Liegen die Ursachen der Arbeitslosigkeit jedoch jenseits der eigenen Kontrolle (sei es aufgrund von Krankheit oder der Lage auf dem Arbeitsmarkt), wird dieser Anspruch akzeptiert.

Diese Aussage wird durch empirische Untersuchungen unterstützt, die belegen, daß die meisten Menschen zwar jenen helfen möchten, die unverschuldet in eine Notsituation geraten, aber die Hilfe für jene ablehnen, die durch eigenes Verschulden in eine unvorteilhafte Situation gekommen sind. Verschiedene empirische Untersuchungen zusammenfassend schreibt Wax (2000, S. 274): „The data suggest that a majority of voters favor collective responsibility for the poor and want the poor become economically independent. They are reluctant, however, to support needy persons who show little interest in supporting themselves". In diesem Sinne äußern sich auch Bowles und Gintis (1998): ,... policies that reward people independent of whether and how much they contribute to society are considered unfair and are not supported, even if the intended recipients are otherwise worthy of support, and even if the incidence of noncontribution in the target population is rather low" (S. 15). ${ }^{21}$

\footnotetext{
${ }^{21}$ Die Aussage von Bundeskanzler Gerhart Schröder, wonach es "kein Recht auf Faulheit" gebe, geht in die gleiche Richtung. Arbeitslosenhilfe für unverschuldete Arbeitslosigkeit wird ebenso als "fair" beurteilt, wie es als unsolidarisch und unfair gilt, wenn jemand Leistungen erhält, obwohl es in der eigenen Kontrolle der betreffenden Person läge, eine Arbeit anzunehmen.
} 
Selbstverständlich ist es im Detail schwierig zu sagen, was selbstverschuldet bzw. jenseits der eigenen Kontrolle ist (vgl. Wax 2000 für eine Diskussion). Dies dürfte nicht zuletzt von kulturellen und sozialen Erwartungen abhängen. Dennoch bleibt festzuhalten, daß sozialstaatliche Arrangements um so mehr Akzeptanz und Unterstützung erhalten, je mehr der Grundsatz gilt, daß nur der Leistungen erhält, der sich zuvor im Rahmen seiner Möglichkeiten selbst bemüht hat. Diese Einsicht ist auch relevant für die politische Umsetzung neuer politischer Programme: Ansätze, die darauf abzielen, die eigene Leistungsfähigkeit und Selbsthilfe bzw. die (Wieder-)Eingliederung in den Arbeitsmarkt zu erhöhen, können mit einer größeren Zustimmung rechnen als reine Umverteilungsprogramme (,workfare statt wellfare“). Beispiele für erstere sind Ausbildungsförderung, Unterstützung bei der Kinderbetreuung, Berufsberatung, Teilzeitarbeitsregelungen, Transporthilfen usw.

Die Akzeptanz sozialpolitischer Maßnahmen kann auch dadurch erhöht werden, daß Transferempfänger nicht nur erhalten, sondern auch etwas zurückgeben. Reziprozität als bedingte Kooperation bedeutet, daß in einem kollektiven Transfersystem Geber viel eher bereit sind, einen großzügigen Beitrag zu leisten, wenn sie das Gefühl haben, daß sie zumindest symbolisch etwas zurückerhalten (s. Prinzipien 1 u. 3). Für die Ausgestaltung sozialpolitischer Maßnahmen folgt hieraus, daß jeder Empfänger (zumindest symbolisch) auch etwas zurück gibt.

Ein Anwendungsbeispiel ist die Asylpolitik. Aus gutem Grund gilt die Gewährung politischen Asyls in der Bundesrepublik als ein zentrales politisches Grundrecht. Dennoch ist die Akzeptanz dieses Grundsatzes erheblichen Schwankungen unterworfen. Wenn es das Ziel der Asylpolitik ist, die Akzeptanz gegenüber Fremden und Asylanten zu stärken und Widerstände gegen das Grundrecht auf politisches Asyl zu verringern, dann ergibt sich aus dem oben Gesagten, daß es sinnvoll ist, Asylanten eine (beschränkte) Arbeitserlaubnis zu erteilen (wie es jetzt in der Bundesrepublik wieder diskutiert wird) bzw. zu geringfügigen Arbeiten (z.B. im sozialen oder ökologischen Bereich) zu verpflichten. Asylsuchende würden hierdurch zumindest teilweise in die Lage versetzt, ihren eigenen Lebensunterhalt zu bestreiten, und würden gleichzeitig signalisieren, daß sie der Gesellschaft ,etwas zurückgeben“. Beides könnte geeignet sein, die Akzeptanz gegenüber der Asylpolitik zu erhöhen. Ähnliche Argumente gelten auch für andere sozialpolitische Maßnahmen und andere Gruppen von Transferbeziehern. ${ }^{22} \mathrm{Um}$ nicht missverstanden $\mathrm{zu}$ werden: Es kann sich selbstverständlich niemals um „,materiell gleichberechtigte“ Gegenleistungen halten, da es sich ja gerade um sozialpolitisch motivierte Transferleistungen handelt. Wenn aber die Geber realisieren, daß auch die Empfänger ihren Beitrag leisten - und sei er auch gering - steigt die Bereitschaft zu teilen.

Der Grundsatz, wonach jemand, der etwas erhält auch etwas zurück geben sollte, erhöht nicht nur die Akzeptanz staatlicher Transferleistungen, sondern verringert auch die Schamgefühle der Empfänger. ${ }^{23}$ Schamgefühle erklären, warum in einem erheblichen Ausmaß soziale Leistungen, auf die ein Anspruch bestünde, nicht

22 Ein ähnliches Argument lässt sich auch im Hinblick auf die Zustimmung zum gesetzlichen Rentensystem anbringen. Vielfach wird die „Aufkündigung des Generationenvertrages“ beklagt. Wenn das System der gesetzlichen Rentenversicherung nicht verhindern kann, daß jüngere Menschen wesentlich mehr einzahlen, als sie später an Rentenleistungen zurückerhalten, ist die mangelnde Bereitschaft, sich dem gesetzlichen Rentensystem anzuvertrauen, nicht verwunderlich. Der Homo Reciprocans achtet auf einen fairen Ausgleich der Lasten - auch über die Zeit.

23 In der soziologischen Literatur wurde die Stigmatisierung von Transferempfängern häufig dokumentiert vgl. z.B. Horan and Austin (1974). 
angenommen werden (Moffitt 1983). ${ }^{24}$ Gleichzeitig gilt, daß vor allem solche Leistungen in Anspruch genommen werden, bei denen die Leistungsempfänger bereits Ansprüche durch eigene Beiträge erworben haben. Es handelt sich also um Leistungen, bei denen die Empfänger im Voraus eine Gegenleistung erbracht haben. In diesem Sinne stellt Lindbeck (1995) fest: ,... individuals are less hesitant to live on, and adjust their lives to, general social security benefits... . After all, such benefits are often described today as "citizen rights" and "entitlements", based on contributions paid previously (Hervorhebung durch AF)" (S. 480-481).

\subsection{Anreizverträge und "Hidden Costs of Incentives"}

Die Forderung nach Einführung von Anreizverträgen in Firmen und öffentlichen Verwaltungen ist in der öffentlichen Diskussion en vogue. Die Intuition für explizite Leistungsanreize ist einfach und bestechend. Es ist ein Standardergebnis der PrinzipalAgenten-Literatur, daß durch die geeignete Wahl expliziter Anreize die Arbeitsleistung von Agenten systematisch erhöht werden kann. Wie im Experiment in Abschnitt 3.4 gezeigt wurde, können allerdings auch durch Fairness und Reziprozität zumindest teilweise Qualitäts- bzw. Leistungsniveaus durchgesetzt werden, die in der Allgegenwart eigennütziger Individuen nicht zu erwarten wären. Es stellt sich somit die Frage, wie explizite Anreize mit Reziprozität interagieren. Eine Möglichkeit besteht darin, daß sie hinzuaddiert werden können, d.h. daß Reziprozität und explizite Anreize sich gegenseitig ergänzen. Es ist allerdings auch möglich, daß die Einführung expliziter Anreize das Vertrauensklima, das die Voraussetzung einer reziprozitätsbedingten Leistungsmotivation bildet, unterminiert. In diesem Falle entstehen durch die Einführung expliziter Anreize Kosten, die von der auf dem Homo Oeconomicus Modell basierenden Prinzipal-Agenten Literatur bislang nicht reflektiert wurde.

In der Tat konnte in Experimenten gezeigt werden, daß explizite Leistungsanreize freiwillige Formen von Kooperation verdrängen können (Fehr und Gächter 2000b). In Experimenten, die dem in Abschnitt 3.4 vorgestellten sehr ähnlich waren, führte die Einführung von expliziten Anreizen statt zu einer erhöhten sogar zu einer geringeren Effizienz. Während in einer Institution ohne explizite Anreize Käufer in der Lage waren, durch „faire“ Preise höhere Qualitäten seitens der (reziproken) Verkäufer zu erzielen, gelang dies in einem Regime mit expliziten Anreizen nicht mehr. Offenbar führte die Einführung expliziter Anreize zu einer Verschlechterung der Atmosphäre, die wiederum zu einem Zusammenbruch der positiven Beziehung zwischen Preis und Qualität führte (vgl. Figur 4). Andere Studien, die auf dysfunktionale Tendenzen expliziter Anreize verweisen, sind Bohnet, Frey und Huck (1999), Frey (1997a) und Gneezy und Rustichini (2000)) sowie aus der Sicht der Sozialpsychologie Deci und Ryan (1985). Der Tenor der zitierten Arbeiten lautet, daß die unkritisch geforderte Einführung von expliziten Anreizen problematischer ist, als es die Standardperspektive suggeriert. Die Aufhebung der Annahme, alle Individuen verhielten sich wie der Homo Oeconomicus, rückt potentielle Kosten in Form verdrängter freiwilliger Kooperationsformen und verringertem gegenseitigem Vertrauen ins Blickfeld. Es ist deshalb im Einzelfall genau zu prüfen, ob die Einführung von Anreizmechanismen diese Kosten rechtfertigt (vgl. Gächter und Falk 2000 für eine Diskussion verschiedener Anreizmechanismen).

\footnotetext{
${ }^{24}$ Ein solcher Zuwendungsverzicht steht ebenso im Widerspruch zum Konzept des Homo Oeconomicus, wie die Tatsache, daß weniger Steuern hinterzogen werden, als es (bei Geltung der Eigennutzhypothese) rational wäre (s.o.).
} 


\subsection{Kriminalität als Soziale Interaktion: Die Rolle von Belief-Management}

Für kriminelles Verhalten gilt in den Wirtschaftswissenschaften beinahe einhellig das Beckersche Diktum, wonach ersteres einer rationalen Kosten-Nutzen-Überlegung entspringt: Solange die materiellen Kosten, die mit einer kriminellen Handlung verbunden sind, den Nutzen übersteigen, wird ein rationales Individuum sich regelkonform verhalten (Becker 1968).

Die aus der Existenz des Homo Reciprocans abgeleitete alternative Hypothese versteht Kriminalität hingegen zusätzlich als eine Form bedingten Verhaltens: Wenn andere in meiner Umgebung sich nicht regelkonform verhalten, steigt auch meine Bereitschaft zur Nichteinhaltung von Regeln (s. Prinzip 3). Der wesentliche Punkt hierbei ist, daß die Entscheidung zur kriminellen Handlung nicht alleine von einer Abwägung materieller Faktoren abhängt, sondern auch von den Erwartungen über das Verhalten anderer in meiner Umgebung. Regelkonformes Verhalten ist aus dieser Perspektive ein soziales Interaktionsphänomen: Wenn jemand die Erwartung hegt, in seiner Umgebung verhalten sich die anderen pro-sozial, wird er sich ceteris paribus ebenso verhalten und vice versa. Aus dieser Überlegung folgt unmittelbar, daß es ,gute“ und „schlechte“ Erwartungsgleichgewichte gibt. In ersteren führt die Erwartung, daß sich alle an die Regeln halten, in der Tat dazu, daß die Regeln eingehalten werden, während in letzteren die Erwartung von Regelverstößen zur Erosion von Regeln führt. Evidenz für diese Form bedingter Regelkonformität wurde in zahlreichen Studien belegt (vgl. Kahan 1997 oder Glaeser, Sacerdote und Scheinkman 1996).

Wie bereits erwähnt, ergibt sich aus der Existenz von guten und schlechten Erwartungsgleichgewichten für die Politik durch geeignetes Belief-Management ein interessantes Politik-Instrument. Ein möglicher Ansatzpunkt für politisches BeliefManagement ist die Gestaltung des äußeren Erscheinungsbildes von öffentlichen Plätzen und Einrichtungen. Während ein intaktes Erscheinungsbild die Geltung sozialer Normen und Regeln unterstreicht, führt ein desolater Zustand öffentlicher Räume zur (sich selbst erfüllenden) Erwartung weiterer Verwahrlosung (vgl. das sogenannte „broken window“-Phänomen). In seiner empirischen Untersuchung in 40 US Amerikanischen Städten belegt Skogan (1990) einen positiven Zusammenhang zwischen ,public disorder" in den entsprechenden Vierteln und der Kriminalitätsrate. Kahan (1997) stellt in diesem Zusammenhang auf den potentiellen Nutzen einer Politik $\mathrm{ab}$, die die Verwahrlosung öffentlicher Räume unterdrückt, da sichtbare Formen von Regelverstößen zu weiteren kriminellen Handlungen ermuntern und anstiften.

Der Zusammenhang zwischen der äußeren Erscheinung öffentlicher Räume und der Bereitschaft, Normen und Regeln einzuhalten, wurde eindrucksvoll in einem Feldexperiment von Cialdini, Reno und Kallgren (1990) nachgewiesen. In ihrer Studie testen sie, ob Individuen vermehrt Abfälle in einer Umgebung wegwerfen, in der bereits viel Müll „herumliegt“. In einem ihrer Experimente heften sie in einem Parkhaus Informationszettel $^{25}$ an die Windschutzscheibe der abgestellten Autos. In der ersten Experiment-Bedingung ist das Parkhaus ,sauber“, in der zweiten ,verschmutzt“. Es stellt sich heraus, daß in einem sauberen Parkhaus wesentlich weniger Informationszettel weggeworfen werden als im bereits verschmutzten. Dies zeigt, daß die Umgebung ein starkes Signal über die Geltung und Einhaltung von Normen und Regeln aussendet, auf das viele Menschen bedingt reagieren: Wenn andere ihren Abfall

\footnotetext{
${ }^{25}$ Auf diesen Zetteln stand: „This is Automotive Safety Week. Please drive carefully“.
} 
wegwerfen, tun sie es auch. Herrscht hingegen der Eindruck einer intakten Regelbefolgung vor, verhalten sich viele entsprechend dieser Norm.

Für politisches Belief-Management folgt hieraus, daß der Eindruck allgemeiner Regelbefolgung vermittelt werden sollte. Es lohnt sich, zur Bekämpfung von KleinKriminalität für ein entsprechendes Erscheinungsbild öffentlicher Räume zu sorgen und die öffentliche Infrastruktur in einer Form zu präsentieren, die zu ,,positiver Reziprozität einlädt". In diesem Zusammenhang kann auch die sogenannte ,Zero tolerance“-Politik, die v.a. in New York angewendet wurde, gesehen werden. Die konsequente Bestrafung selbst geringfügiger Delikte hat demnach mehrere Effekte. Zum einen entfaltet sie eine Abschreckungswirkung, ganz im Einklang zum standard-ökonomischen Kalkül. Zweitens führt sie aufgrund der erhöhten Abschreckung zu der Einschätzung, daß insgesamt weniger gegen Regeln verstoßen wird, was gemäss Prinzip 3 allein schon zu weniger bedingten Regelverstößen führt. Schließlich führt eine Politik, die auch Sachbeschädigung etc. bekämpft, zu einem verbesserten Erscheinungsbild, unterstreicht die Geltung und Durchsetzung von Normen und führt somit zu einer Koordinierung auf "gute Erwartungsgleichgewichte“.

\subsection{Politik der ,kleinen Einheiten“}

Aus den in dieser Arbeit diskutierten Verhaltensprinzipien ergeben sich auch neue Gesichtspunkte für die Debatte um Subsidiarität, Bürgergesellschaft und eine Politik der „kleinen Einheiten“. Wie dargelegt, verfügen Gesellschaften über informelle, auf Fairness und Reziprozität basierende Sanktionsverfahren. Es wurde gezeigt, daß reziproke Individuen Trittbrett-Fahren bestrafen und hierdurch Egoisten effektiv disziplinieren. Hierdurch wiederum kann das Beitragsniveau zu öffentlichen Gütern dramatisch erhöht werden (s. Prinzip 5). Diese freiwillige Sanktionierung von individuellem Trittbrettfahrerverhalten setzt aber die Beobachtbarkeit von Fehlverhalten voraus. Diese Voraussetzung ist typischerweise nur in kleineren (politischen) Einheiten vorhanden (Gemeinden, Quartiere, Vereine). Im Sinne einer Bekämpfung von illegitim in Anspruch genommenen Transferleistungen und der verbesserten Bereitstellung lokaler öffentlicher Güter, könnte es sich demnach lohnen, kleine Einheiten politisch aufzuwerten und ihnen in höherem Maße Einnahme- und Ausgabekompetenzen zu übertragen.

Dieses Argument wird noch verstärkt durch die Tatsache, daß die Mitglieder kleinerer politischer Einheiten häufig wiederholt miteinander interagieren. Es wurde in zahlreichen Experimenten nachgewiesen, daß sich wiederholte Interaktionen positiv auf das Beitragsverhalten in öffentlichen Gut-Situationen auswirken (Keser und van Winden 2000). Gächter und Falk (1999) haben gezeigt, daß in einer vertraglich unvollständigen Tauschsituation langfristige Beziehungen zu mehr Reziprozität und höherer Effizienz führen als einmalige Interaktionen. Auch theoretisch wurde gezeigt, daß sich wiederholte Interaktionen günstig auf die Bereitstellung lokaler öffentlicher Güter auswirkt (z.B. Bowles 1999). Hieraus folgt, daß kleinere politische Einheiten besser in der Lage sind, öffentliche Güter bereitzustellen und Defekteure effektiv zu bestrafen. Dies macht kleine Einheiten für die Übernahme politischer Aufgaben attraktiv.

\subsection{Konsequenzen für den Arbeitsmarkt}

Prinzip 4 besagt, daß die Existenz des Homo Reciprocans aggregierte Marktergebnisse auf vertraglich vollständigen Märkten kaum, auf unvollständigen Märkten jedoch sehr wohl beeinflussen kann. Das wichtigste Beispiel ist der Arbeitsmarkt. Es gilt heute als 
unumstritten, daß der Arbeitsvertrag in hohem Masse unvollständig ist und die exakten Aufgaben, die sich im Verlaufe einer Arbeitsbeziehung ergeben, nur rudimentär spezifiziert (vgl. z.B. Milgrom and Roberts 1992). ${ }^{26}$ Die Unvollständigkeit des Arbeitsvertrages wiederum verursacht ein Motivationsproblem, da Arbeitnehmer einen erheblichen diskretionären Spielraum über die zu leistende Arbeit besitzen. Eine Möglichkeit, das sich hieraus abgeleitete Motivationsproblem zu verringern, besteht in der Zahlung „fairer“ Löhne. Dies ist das klassische Effizienzlohnargument: Arbeitgeber können durch die Bezahlung „fairer“ Löhne die Arbeitnehmer dazu veranlassen, eine „faire“ Arbeitsleistung zu leisten, wobei „fair" eine Arbeitsleistung meint, die über die gerichtlich durchsetzbare Arbeitsleistung hinausgeht. Für eine solche Fairnessbeziehung sprechen experimentelle Laboruntersuchungen (vgl. Fehr, Kirchsteiger und Riedl (1993), Fehr und Falk (in Abschnitt 3.4) aber insbesondere auch zahlreiche Befragungsstudien mit personalverantwortlichen Managern (vgl. Agell und Lundborg 1995, Bewley 1999, Campbell und Kamlani 1997). Die Mehrheit der in diesen Studien befragten Personalmanager antwortet auf die Frage, warum sie - obwohl sie dies aufgrund der Marktsituation könnten - nicht die Löhne senken, daß Lohnsenkungen als unfair empfunden würden. Dies wiederum könne die Arbeitsmoral zerstören und zu Leistungszurückhaltung führen.

Die Beurteilung von Lohnsenkungen (und anderer ähnlicher Benachteiligungen der Arbeitnehmer) ist indes nicht völlig unabhängig von der Situation der Firma. Wenn Lohnsenkungen ultima ratio sind, weil beispielsweise ein drohender Konkurs abgewendet werden muss, wenn mit anderen Worten, die Geschäftsleitung „keine andere Wahl" hat, werden diese Maßnahmen als weniger unfair wahrgenommen, als bei Lohnsenkungen, die einen reinen Umverteilungscharakter haben (vgl. Prinzip 2). Es ist deshalb nicht verwunderlich, daß bei Tarifauseinandersetzungen die „Verantwortung“ für unpopuläre Maßnahmen gerne ausländischen Konkurrenten, ungünstigen Marktbedingungen u.s.w. zugeschrieben wird.

Die fairnessbedingt hohen Kosten von Lohnsenkungen zwingen Firmen häufig dazu, Löhne auf indirektem Wege zu kürzen, beispielsweise durch outsourcingAktivitäten (Bewley 1999). Fairnessüberlegungen spielen folglich bei Entscheidungen zur vertikalen Integration von Unternehmen eine wichtige Rolle. Mehr noch: Fairness liefert eine mögliche Erklärung für die auf Arbeitsmärkten beobachteten Lohnstarrheiten. Agell (1999) führt in einer Übersicht über verschiedene empirische Studien aus: “... persistent social norms are an independent and important cause of wage rigidity..." (S. 144). Es sind in diesem Fall keine staatlichen Regulierungen, die Rigiditäten hervorrufen, sondern freiwillige und dezentrale Vereinbarungen von Firmen und Arbeitnehmern. Selbst wenn es gelänge, alle staatlichen Regulierungen abzuschaffen, ergeben sich auf unvollständigen Vertragsmärkten dennoch endogen, d.h. allein aus dem Zusammenspiel von Marktteilnehmern, Rigiditäten. Diese wiederum können $\mathrm{zu}$ unfreiwilliger Arbeitslosigkeit und nicht-kompensierenden Lohndifferentialen führen (vgl. Akerlof 1982, Akerlof und Yellen 1990).

\footnotetext{
26 "The employment contract is typically quite imprecise. The employees agree that - within limits that are rarely completely described and only partly understood - they will use their minds and muscles to undertake the tasks that the employer directs them to do. The employer agrees to pay the employees. The range of actions that might be requested or required is unclear" (Milgrom and Roberts 1993, S. 329).
} 


\section{Schlussbemerkungen}

Ökonomische Theorien und Handlungsvorschläge prägen das öffentliche Bewusstsein sowie politisches und unternehmerisches Handeln. Kaum ein anderes erkenntnis- und handlungsleitendes Modell wie das des Homo Oeconomicus hat daher einen vergleichbaren politischen Einfluss. Durch die Entwicklung experimenteller Methoden ist es möglich, die Annahmen des Homo Oeconomicus Modells unter kontrollierten Laborbedingungen mit dem tatsächlichen Handeln von Individuen zu vergleichen. Hierbei zeigt sich, daß der Homo Oeconomicus weitaus weniger universell ist, als gemeinhin angenommen. Empirisch stellt sich heraus, daß - obwohl der Homo Oeconomicus existiert - die Mehrheit der Experimentalteilnehmer faires Verhalten belohnt und unfaires Verhalten bestraft (Reziprozität).

Einem Missverständnis soll hier Vorschub geleistet werden: Reziprozität hat nichts mit Altruismus oder Güte zu tun. Reziproke Individuen sind nicht immer gut und rechtschaffend, sondern verhalten sich nur bei entsprechenden Erwartungen bzw. einem entsprechenden Umfeld kooperativ. Es soll auch nicht behauptet werden, der Homo Oeconomicus sei „schlecht“. Es geht in der vorliegenden Arbeit lediglich darum, Verhaltensregularitäten zu beschreiben und ihre Bedeutung für die Wirtschafts- oder Sozialpolitik anzudeuten. Es gibt Situationen, in denen Gesellschaften „erfolgreicher“ wären, wenn sie nur aus Egoisten bestünden. Rache und Vergeltung, sei es während eines Krieges zwischen Kämpfern verfeindeter Länder oder zwischen Clans oder Privatpersonen können zerstörerisch und ineffizient sein. Nur reziproke Individuen lassen sich auf derartige Negativspiralen ein, Egoisten nicht. Zudem muss betont werden, daß kooperatives Verhalten einer Gruppe nachteilig für andere Gruppen sein kann. Es mag sein, daß informelle Sanktionen und bedingte Kooperation eine Kollusion auf Gütermärkten befördert, was zwar gut für die Kartellmitglieder aber zugleich schädlich für die Verbraucher ist. Auch gilt, daß eine Firma, die „faire“ Löhne zahlt, um höhere Arbeitsleistungen $\mathrm{zu}$ erhalten, sich und „ihre“ Arbeitnehmer besser stellt. Gleichzeitig kann ein solcher „Effizienzlohn“ jedoch zur Existenz unfreiwilliger Arbeitslosigkeit führen. Schließlich sei darauf hingewiesen, daß die traditionelle Analyse vollständiger Wettbewerbsmärkte durch die Existenz von Reziprozität nicht in Frage gestellt wird. Unterschiedliche Prognosen werden allerdings auf Märkten mit unvollständigen Verträgen erwartet. Der Arbeitsmarkt ist hierfür das prominenteste Beispiel (s. Abschnitt 4.6).

Die zentrale These dieser Arbeit ist, daß das Modell des Homo Oeconomicus wesentliche Determinanten menschlichen Verhaltens unberücksichtigt lässt und dadurch Gefahr läuft, falsche oder zumindest unvollständige Politikempfehlungen zu fundieren. Das bedeutet nicht notwendigerweise eine vollständige Umkehrung vorhandener Grundsätze, zumindest aber deren substantielle Erweiterung. Die vorliegende Arbeit skizziert einige Implikationen, die sich aus der Existenz des Homo Reciprocans ergeben:

- Aus der Existenz des Homo Reciprocans folgt, daß Gesellschaften und soziale Beziehungen über informelle Mechanismen zur Durchsetzung von Normen und Regeln verfügen, die mit den Annahmen des Homo Oeconomicus nicht erklärbar sind. Diese zu formalen Durchsetzungsmechanismen komplementären Mechanismen bilden einen wesentlichen Teil des Sozialkapitals einer Gesellschaft.

- Dieses Sozialkapital kann durch Politik genutzt und verstärkt werden. Beispiele hierfür sind die Fairness des Steuersystems oder verstärkte politische 
Partizipationsrechte. Gleichzeitig gilt, daß Normen formale Regeln nicht nur ergänzen, sondern daß Politik auch einen Einfluss auf soziale Normen hat: In diesem Sinne gilt es zu bedenken, daß durch Politikmaßnahmen Sozialkapital unterminiert werden kann. ${ }^{27}$

- Da der Homo Reciprocans ein bedingtes Verhalten aufweist, spielen seine Erwartungen und Einschätzungen über das Verhalten anderer eine zentrale Rolle. Das Belief-Management dieser Erwartungen (z.B. über die Gestaltung öffentlicher Plätze oder auch des Steuer- und Rechtssystems) ist ein interessanter Ansatzpunkt für eine alternative Politik.

- Sozialstaatliche Arrangements können sich einer breiten Akzeptanz versichern, wenn fundamentale Fairnessgrundsätze nicht verletzt werden und Leistungen an den Willen zur Selbsthilfe geknüpft werden. Hieraus folgt, daß staatliche Hilfen nicht nur am Bedarf, sondern auch daran ausgerichtet werden, ob eine Verbesserung der Situation außerhalb der Kontrolle der betreffenden Person liegt.

- Reziprozität ermöglicht eine gewisse Vertragsdurchsetzung bei unvollständigen Verträgen. Explizite Anreizverträge können diese freiwilligen Formen der Durchsetzung u.U. unterminieren. Es müssen deshalb die Vorteile einer Einführung expliziter Anreizsysteme mit diesen ,hidden costs of incentives“ abgewogen werden.

- Die Nutzung informeller Sanktionen als eines Mechanismus, der die Bereitstellung lokaler öffentlicher Güter vereinfacht, ist an die Beobachtbarkeit individuellen Verhaltens geknüpft und insbesondere in kleineren politischen Einheiten möglich. Es könnte sich demnach lohnen, kleine Einheiten politisch aufzuwerten und ihnen vermehrt Einnahme- und Ausgabekompetenzen zu übertragen.

- Reziprozität kann erklären, warum es auf Arbeitsmärkten, unabhängig von staatlichen Eingriffen, zu Lohnstarrheiten, nicht-kompensierenden Lohndifferentialen und unfreiwilliger Arbeitslosigkeit kommen kann.

Selbstverständlich ist mit diesen Ausführungen allenfalls ein erster Schritt getan. Weitere theoretische und empirische Arbeiten sind notwendig, um die Konsequenzen, die sich aus der Existenz reziproken Verhaltens für die politische Diskussion ergeben, herauszuarbeiten.

\footnotetext{
${ }^{27}$ Dies ist auch eine Schlussfolgerung des Überblicksartikels über die Durchsetzung formaler Regeln von Polinsky und Shavell (2000): „There is an emerging literature on social norms that seems worth amplifying because of the influence that social norms have on behavior and supplement to formal laws, and also because of the possibility that laws themselves might influence social norms" (S. 73). Zur Interaktion von sozialen Normen und Institutionen und der Möglichkeit der Unterminierung von Sozialkapital durch politisches Handeln s. auch Bowles (1998), Frey (1992), Lindbeck, Nyberg und Weibull (1999) und Bar-Gill und Fersthman (2000).
} 


\section{Literatur}

Abbink, K., Irlenbusch, B. and Renner, E. (2000): "The Moonlighting Game - An Experimental study on reciprocity and Retribution", Journal of Economic Behavior and Organization 42, 265-277.

Agell, Jonas and Lundborg, Per (1995): "Theories of Pay and Unemployment: Survey Evidence from Swedish Manufacturing Firms", Scandinavian Journal of Economics, vol. 97, 295 - 307.

Agell, J. (1999): "On the Benefits from Rigid Labour Markets: Norms, Market Failures, and Social Insurance", Economic Journal, Vol. 109, pp. F143-F164.

Akerlof, George (1982): Labor Contracts as a Partial Gift Exchange. Quarterly Journal of Economics, 97, 543-569.

Akerlof, George A. and Yellen, Janet L. (1990): "The Fair Wage- Effort Hypothesis and Unemployment", Quarterly Journal of Economics, vol. 105, 255-283.

Alm, J. und Jackson, B. (1993): "Fiscal Exchange, Collective Decision Institutions, and Tax Compliance", Journal of Economic Behavior and Organization 22, 285-303.

Alm, J., McClelland und Schulze, W. (1999): "Changing the Social Norm of Tax Compliance by Voting", Kyklos 52, 141-171.

Andreoni, J., Erard, B. und Feinstein, J. (1998): „Tax Compliance“, Journal of Economic Literature XXXVI, 818-860.

Bar-Gill, Oren und Fersthman, C. (2000): „The Limit of Public Policy: Enodgenous Preferences“, Working Paper, Tel-Aviv University.

Becker, G. S. (1968): „Crime and Punishment: An Economic Approach“, Journal of Political Economy 76, 169-217.

Bewley, Truman (1999): Why Wages Don't Fall During a Recession. Cambridge: Harvard University Press.

Bohnet, I, Huck, S. and Frey, B.S. (1999): "More Order with Less Law: On Contract Enforcement, Trust, and Crowding, Working Paper, Harvard University.

Bolle, F. (1998): "Rewarding Trust - An Experimental Analysis" Theory and Decision 45, 83-98.

Bolle, F. and Kritikos, A. (1998): "Self-Centered Inequality Aversion versus Reciprocity and Altruism", mimeo, 14/95, Europe-University Viadrina, Frankfurt/Oder.

Bowles, S. (1998): „Endogenous Preferences: The Cultural Consequences of Markets and other Economic Institutions", in: Journal of Economic Literature, 75-111.

Bowles, S., and Gintis, H. (1998): "Is Equality Passé? Homo Reciprocans and the Future of Egalitarian Politics", Working Paper, Department of Economics, University of Massachusetts.

Bowles, S. (1999): 'Social Capital' and Community Governance, Working Paper, Department of Economics, University of Massachusetts.

Campbell, C. M. and Kamlani, K. (1997): "The Reasons for Wage Rigidity: Evidence from a Survey of Firms", Quarterly Journal of Economics 112, 759-789.

Deci, E. and Ryan, R. (1985): Intrinsic Motivation and Self-Determination in Human Behavior, New York and London: Plenum Press.

Davis, D.D. und C.A. Holt (1993): Experimental Economics, Princeton.

Falk, A., Fehr, E. und Fischbacher, U. (1999): "On the Nature of Fair Behavior, erscheint in: Economic Inquiry.

Falk, A., Fehr, E. und Fischbacher, U. (2000a): "Informal Sanctions", Working Paper No. 59, Institut für Empirische Wirtschaftsforschung, Universität Zürich. 
Falk, A., Fehr, E. und Fischbacher, U. (2000b): "Testing Theories of Fairness Intentions Matter", Working Paper No. 63, Institut für Empirische Wirtschaftsforschung, Universität Zürich.

Falk, A. und Fischbacher, U. (1999): „A Theory of Reciprocity“, Working Paper No. 6, Institut für Empirische Wirtschaftsforschung, Universität Zürich.

Falk, A. und Fischbacher, U. (1999): "The Economics of Reciprocity - Theory and Evidence", erscheint in: Inequality Around the World: Where Are We and Where Are We Headed, hrsg. von Richard Freeman, Palgrave

Falk, A. und Fischbacher, U. (2000): „Detecting Social Interactions”, geschrieben für die Präsentation in der eingeladenen Session über „Soziale Normen“ des 2001 meetings der European Economic Association.

Falk, A. und Tyran, J.R. (1997): „Experimentelle Wirtschaftsforschung“, in: Wirtschaftswissenschaftliches Studium 6, 326-329.

Fehr, E. und Falk, A. (1999): „Wage Rigidity in a Competitive Incomplete Contract Market“, Journal of Political Economy 107, 106-134.

Fehr, E. und Gächter, S. (2000a): „Fairness and Retaliation: The Economics of Reciprocity“, Journal of Economic Perspectives 14, 159-181.

Fehr, E. und Gächter, S. (2000b): „Do Incentive Contracts Crowd-Out Voluntary Cooperation?", Working Paper No. 34, Institut für Empirische Wirtschaftsforschung.

Fehr, E. und Gächter, S. (2000c): "Cooperation and Punishment in Public Good Experiments - An Experimental Analysis of Norm Formation and Norm Enforcement", American Economic Review 90, 980-994.

Fehr, Ernst, Georg Kirchsteiger, and Arno Riedl (1993): Does Fairness prevent Market Clearing? An Experimental Investigation. Quarterly Journal of Economics, 108, 437-460.

Fehr, E. und Tougareva, E. (1995): „Do High Stakes Remove Reciprocal Fairness Evidence from Russia”, Discussion paper, Institut für Empirische Wirtschaftsforschung, Universität Zürich.

Feld, L. und Frey, B. S. (2000): „Trust Breeds Trust: How Taxpayers are Treated“, erscheint in: Economies and Governance.

Frey, B. S. (1992): “Tertium Datur: Pricing, Regulating and Intrinsic Motivation", Kyklos 45, 161-184.

Frey, B. S. (1997): “A Constitution for Knaves Crowds out Civic Virtues”, The Economic Journal 107, 1043-1053.

Frey, B. S. (1993): "Does Monitoring Increase Work Effort? The Rivalry with Trust and Loyalty", Economic Inquiry 31, 663-670.

Frey, B. S. (1997a): Markt und Motivation - Wie ökonomische Anreize die (Arbeits-) Moral verdrängen, München: Verlag Vahlen.

Gneezy, U. und Rustichini, A. (1999): “A Fine is a Price”, erscheint in: Journal of Legal Studies.

Friedman, Daniel, Rust, J. (eds.) (1993): “The Double Auction Market: Theories and Evidence", Santa Fe Institute Series in the Science of the Complexity, Proceedings vol. 15. Reading, Mass.: Addison-Wesley.

Friedman, D. and S. Sunder (1994): Experimental Methods: A Primer for Economists, Cambridge. 
Gächter, S. und Falk, A. (1999): „Reputation and Reciprocity: Consequences for the Labour Relation", erscheint in: Scandinavian Journal of Economics

Gächter, S. und Falk, A. (2000): „Work Motivation, Institutions, and Performance”, erscheint in: Advances in Experimental Business Research, hrsg. von Rami Zwick und Amnon Rapoport, Kluwer Academic Publishers.

Goette, L. und Kucher, M. (1998): „Trust Me. An Empirical Analysis of Taxpayer Honesty“, in: Finanzarchiv 55, 429-444.

Güth, W., Schmittberger, R. and Schwarze, B. (1982): “An Experimental Analysis of Ultimatium Bargaining", Journal of Economic Behavior and Organization 3, 36788.

Glaeser, E., Sacerdote, B. und Scheinkman J. (1996): „Crime and Social Interactions“, Quarterly Journal of Economics 111, 507-548.

Gouldner, A. (1960): "The Norm of Reciprocity", American Sociological Review 25, $161-178$

Horan, P. and Austin, P. (1974): "The Social Bases of Welfare Stigma", in: Social Problems 21, 648-657.

Hechter, M. (1987): Principles of Group Solidarity, The University of California Press.

Jacobsen, E. and Sadrieh, A. (1996): "Experimental Proof for the Motivational Importance of Reciprocity”, Discussion Paper No. B-386, University of Bonn.

Kagel, J.H., A.E. Roth (Hrsg.), The Handbook of Experimental Economics, Princeton 1995.

Kahan, D. (1997): "Social Influence, Social Meaning, and Deterrence", Virginia Law Review 83, 349-395.

Keser, C. und van Winden, F. (2000): "Conditional Cooperation and Voluntary Contributions to Public Goods", in: Scandinavian Journal of Economics 102, 23 39.

Kirchgässner, G. (1991): Homo Oeconomicus: Das ökonomische Modell individuellen Verhaltens und seine Anwendungen in den Wirtschafts- und Sozialwissenschaften, Tübingen: Mohr.

Lind, E. A. und T. R. Tyler (1988) The Social Psychology of Procedural Jusitce, New York: Plenum Press.

Lindbeck, A. (1995): „Welfare State Disincentives with Endogenous Habits and Norms“, in: Scandinavian Journal of Economics 97, 477-494.

Lindbeck, A. Nyberg, S. and Weibull, J. W. (1999): "Social Norms and Economic Incentives in the Welfare State", in: The Quarterly Journal of Economics, Vol. XCIV, 1-35.

Milgrom, Paul and John Roberts (1992): Economics, Organization and Management. New Jersey: Prentice Hall International.

Moffitt, R. (1983): "An Economic Model of Welfare Stigma", American Economic Review 73, 1023-1035.

Polinsky, M. A. und Shavell, S. (2000): "The Economic Theory of Public Enforcement of Law", Journal of Economic Literature XXXVIII, 45-76.

Pommerehne, W., Hart, A. und Frey, B. S. (1995): „Tax Morale, Tax Evasion and the Choice of Policy Instruments in Different Political Systems“, Public Finance and Irregular Activities, Beiheft zu: Public Finance/Finances Publiques 49, 52-69.

Rabin, Matthew (1993): Incorporating Fairness into Game Theory and Economics. American Economic Review, 83(5), 1281 - 1302.

Schneider, F. (2000): "Some Elements of a European Federal Union: A Public Choice Approach", in: Public Choice Analysis of Economic Policy (by K. Alec Chrystal 
and Rupert Pennant-Rea), Proceedings of the British Association for the Advancement of Science, Section F (Economics), Macmillian Press Ltd, Great Britain, 149-170.

Schneider, F., und Enste, D. H. (2000): „Shadow Economies: Size, Causes, and Consequences“, in: Journal of Economic Literature XXXVIII, 77-114.

Seidl, C. und Traub, S. (1999): „Taxpayers' Attitudes, Behavior, and Perception of Fairness in Taxation“, Working Paper, Institut für Finanzwissenschaft und Sozialpolitik, Universität zu Kiel.

Selten, R. (1978): “The Equity Principle in Economic Behavior", In: Decision Theory and Social Ethics. Issues in Social Choice, H. Gottinger and W. Leinfellner (Eds.), Reidel Dordrecht.

Skogan, W. G. (1990): Disorder and Decline: Crime and the Spiral of Decay in American Neighborhoods. University of California Press.

Slemrod, J. (1992): "Why People Pay Taxes: Tax Compliance and Enforcement", University of Michigan Press, Ann Arbor.

Smith, V. (1976): "Experimental Economics: Induced Value Theory, in: American Economic Review, Papers and Proceedings 66, S. 274-279.

Sugden, R. (1986): The Economics of Rights, Co-Operation and Welfare, Oxford, UK: Basil Blackwell.

Trivers, R. (1971): "The Evolution of Reciprocal Altruism", Quarterly Review of Biology 46, 35-57.

de Waal, F. (1996): Good Natured. The Origins of Right and Wrong in Humans and Other Animals. Harvard University Press.

Wax, A. (2000): "Rethinking Welfare Rights: Reciprocity Norms, Reactive Attitudes, and the Political Economy of Welfare Reform", Law and Contemporary Problems 63, 257-298. 


\section{Working Papers of the Institute for Empirical Research in Economics}

No.

1. Rudolf Winter-Ebmer and Josef Zweimüller: Firm Size Wage Differentials in Switzerland: Evidence from Job Changers, February 1999

2. Bruno S. Frey and Marcel Kucher: History as Reflected in Capital Markets: The Case of World War II, February 1999

3. Josef Falkinger, Ernst Fehr, Simon Gächter and Rudolf Winter-Ebmer: A Simple Mechanism for the Efficient Provision of Public Goods - Experimental Evidence, February 1999

4. Ernst Fehr and Klaus M. Schmidt: A Theory of Fairness, Competition and Cooperation, April 1999

5. Markus Knell: Social Comparisons, Inequality, and Growth, April 1999

6. Armin Falk and Urs Fischbacher: A Theory of Reciprocity, July 2000

7. Bruno S. Frey and Lorenz Goette: Does Pay Motivate Volunteers?, May 1999

8. Rudolf Winter-Ebmer and Josef Zweimüller: Intra-firm Wage Dispersion and Firm Performance, May 1999

9. Josef Zweimüller: Schumpeterian Entrepreneurs Meet Engel's Law: The Impact of Inequality on InnovationDriven Growth, May 1999

10. Ernst Fehr and Simon Gächter: Cooperation and Punishment in Public Goods Experiments, June 1999

11. Rudolf Winter-Ebmer and Josef Zweimüller: Do Immigrants Displace Young Native Workers: The Austrian Experience, June 1999

12. Ernst Fehr and Jean-Robert Tyran: Does Money Illusion Matter?, June 1999

13. Stefan Felder and Reto Schleiniger: Environmental Tax Reform: Efficiency and Political Feasibility, July 1999

14. Bruno S. Frey: Art Fakes - What Fakes?, An Economic View, July 1999

15. Bruno S. Frey and Alois Stutzer: Happiness, Economy and Institutions, July 1999

16. Urs Fischbacher, Simon Gächter and Ernst Fehr: Are People Conditionally Cooperative? Evidence from a Public Goods Experiment, July 2000

17. Armin Falk, Ernst Fehr and Urs Fischbacher: On the Nature of Fair Behavior, August 1999

18. Vital Anderhub, Simon Gächter and Manfred Königstein: Efficient Contracting and Fair Play in a Simple Principal-Agent Experiment, September 2000

19. Simon Gächter and Armin Falk: Reputation or Reciprocity? An Experimental Investigation, April 2001

20. Ernst Fehr and Klaus M. Schmidt: Fairness, Incentives, and Contractual Choices, September 1999

21. Urs Fischbacher: $z$-Tree - Experimenter's Manual, September 1999

22. Bruno S. Frey and Alois Stutzer: Maximising Happiness?, October 1999

23. Alois Stutzer: Demokratieindizes für die Kantone der Schweiz, October 1999

24. Bruno S. Frey: Was bewirkt die Volkswirtschaftslehre?, October 1999

25. Bruno S. Frey, Marcel Kucher and Alois Stutzer: Outcome, Process \& Power in Direct Democracy, November 1999

26. Bruno S. Frey and Reto Jegen: Motivation Crowding Theory: A Survey of Empirical Evidence, November 1999

27. Margit Osterloh and Bruno S. Frey: Motivation, Knowledge Transfer, and Organizational Forms, November 1999

28. Bruno S. Frey and Marcel Kucher: Managerial Power and Compensation, December 1999

29. Reto Schleiniger: Ecological Tax Reform with Exemptions for the Export Sector in a two Sector two Factor Model, December 1999

30. Jens-Ulrich Peter and Klaus Reiner Schenk-Hoppé: Business Cycle Phenomena in Overlapping Generations Economies with Stochastic Production, December 1999

31. Josef Zweimüller: Inequality, Redistribution, and Economic Growth, January 2000

32. Marc Oliver Bettzüge and Thorsten Hens: Financial Innovation, Communication and the Theory of the Firm, January 2000

33. Klaus Reiner Schenk-Hoppé: Is there a Golden Rule for the Stochastic Solow Growth Model? January 2000

34. Ernst Fehr and Simon Gächter: Do Incentive Contracts Crowd out Voluntary Cooperation? February 2000

35. Marc Oliver Bettzüge and Thorsten Hens: An Evolutionary Approach to Financial Innovation, July 2000

36. Bruno S. Frey: Does Economics Have an Effect? Towards an Economics of Economics, February 2000

37. Josef Zweimüller and Rudolf Winter-Ebmer: Firm-Specific Training: Consequences for Job-Mobility, March 2000

The Working Papers of the Institute for Empirical Research in Economics can be downloaded in PDF-format from http://www.unizh.ch/iew/wp/ 


\section{Working Papers of the Institute for Empirical Research in Economics}

No.

38. Martin Brown, Armin Falk and Ernst Fehr: Contract Inforcement and the Evolution of Longrun Relations, March 2000

39. Thorsten Hens, Jörg Laitenberger and Andreas Löffler: On Uniqueness of Equilibria in the CAPM, July 2000

40. Ernst Fehr and Simon Gächter: Fairness and Retaliation: The Economics of Reciprocity, March 2000

41. Rafael Lalive, Jan C. van Ours and Josef Zweimüller: The Impact of Active Labor Market Programs and Benefit Entitlement Rules on the Duration of Unemployment, March 2000

42. Reto Schleiniger: Consumption Taxes and International Competitiveness in a Keynesian World, April 2000

43. Ernst Fehr and Peter K. Zych: Intertemporal Choice under Habit Formation, May 2000

44. Ernst Fehr and Lorenz Goette: Robustness and Real Consequences of Nominal Wage Rigidity, May 2000

45. Ernst Fehr and Jean-Robert Tyran: Does Money Illusion Matter? REVISED VERSION, May 2000

46. Klaus Reiner Schenk-Hoppé: Sample-Path Stability of Non-Stationary Dynamic Economic Systems, Juni 2000

47. Bruno S. Frey: A Utopia? Government without Territorial Monopoly, June 2000

48. Bruno S. Frey: The Rise and Fall of Festivals, June 2000

49. Bruno S. Frey and Reto Jegen: Motivation Crowding Theory: A Survey of Empirical Evidence, REVISED VERSION, June 2000

50. Albrecht Ritschl and Ulrich Woitek: Did Monetary Forces Cause the Great Depression? A Bayesian VAR Analysis for the U.S. Economy, July 2000

51. Alois Stutzer and Rafael Lalive: The Role of Social Work Norms in Job Searching and Subjective Well-Being, July 2000

52. Iris Bohnet, Bruno S. Frey and Steffen Huck: More Order with Less Law: On Contract Enforcement, Trust, and Crowding, July 2000

53. Armin Falk and Markus Knell: Choosing the Joneses: On the Endogeneity of Reference Groups, July 2000

54. Klaus Reiner Schenk-Hoppé: Economic Growth and Business Cycles: A Critical Comment on Detrending Time Series, May 2001 - Revised Version

55. Armin Falk, Ernst Fehr and Urs Fischbacher: Appropriating the Commons - A Theoretical Explanation, September 2000

56. Bruno S. Frey and Reiner Eichenberger: A Proposal for a Flexible Europe, August 2000

57. Reiner Eichenberger and Bruno S. Frey: Europe's Eminent Economists: A Quantitative Analysis, September 2000

58. Bruno S. Frey: Why Economists Disregard Economic Methodology, September 2000

59. Armin Falk, Ernst Fehr, Urs Fischbacher: Informal Sanctions, September 2000

60. Rafael Lalive: Did we Overestimate the Value of Health?, October 2000

61. Matthias Benz, Marcel Kucher and Alois Stutzer: Are Stock Options the Managers' Blessing? Stock Option Compensation and Institutional Controls, April 2001

62. Simon Gächter and Armin Falk: Work motivation, institutions, and performance, October 2000

63. Armin Falk, Ernst Fehr and Urs Fischbacher: Testing Theories of Fairness - Intentions Matter, September 2000

64. Ernst Fehr and Klaus Schmidt: Endogenous Incomplete Contracts, November 2000

65. Klaus Reiner Schenk-Hoppé and Björn Schmalfuss: Random fixed points in a stochastic Solow growth model, November 2000

66. Leonard J. Mirman and Klaus Reiner Schenk-Hoppé: Financial Markets and Stochastic Growth, November 2000

67. Klaus Reiner Schenk-Hoppé: Random Dynamical Systems in Economics, December 2000

68. Albrecht Ritschl: Deficit Spending in the Nazi Recovery, 1933-1938: A Critical Reassessment, December 2000

69. Bruno S. Frey and Stephan Meier: Political Economists are Neither Selfish nor Indoctrinated, December 2000

70. Thorsten Hens and Beat Pilgrim: The Transfer Paradox and Sunspot Equilibria, January 2001

71. Thorsten Hens: An Extension of Mantel (1976) to Incomplete Markets, January 2001

72. Ernst Fehr, Alexander Klein and Klaus M. Schmidt: Fairness, Incentives and Contractual Incompleteness, February 2001

73. Reto Schleiniger: Energy Tax Reform with Excemptions for the Energy-Intensive Export Sector, February 2001

74. Thorsten Hens and Klaus Schenk-Hoppé: An Evolutionary Portfolio, May 2001

The Working Papers of the Institute for Empirical Research in Economics can be downloaded in PDF-format from http://www.unizh.ch/iew/wp/

Institute for Empirical Research in Economics, Blümlisalpstr. 10, 8006 Zürich, Switzerland

Phone: 004116343705 Fax: 004116344907 E-mail: bibiewzh@iew.unizh.ch 


\section{Working Papers of the Institute for Empirical Research in Economics}

No.

75. Ernst Fehr and Klaus Schmidt: Theories of Fairness and Reciprocity - Evidence and Economic Applications, February 2001

76. Bruno S. Frey and Alois Stutzer: Beyond Bentham - Measuring Procedural Utility, April 2001

77. Reto Schleiniger: Global $\mathrm{CO}_{2}$-Trade and Local Externalities, April 2001

78. Reto Schleiniger and Stefan Felder: Fossile Energiepolitik jenseits von Kyoto, June 2001

79. Armin Falk: Homo Oeconomicus Versus Homo Reciprocans: Ansätze für ein Neues Wirtschaftspolitisches Leitbild?, July 2001

80. Bruno S. Frey and Alois Stutzer: What can Economists learn from Happiness Research?, May 2001

81. Matthias Benz and Alois Stutzer: Was erklärt die steigenden Managerlöhne? Ein Diskussionsbeitrag, June 2001

82. Peter A.G. VanBergeijk and Jan Marc Berk: The Lucas Critique in Practice: An Empirical Investigation of the Impact of European Monetary Integration on the Term Structure, July 2001

83. Igor V. Evstigneey, Thorsten Hens and Klaus Reiner Schenk-Hoppé: Market Selection of Financial Trading Strategies: Global Stability, July 2001

The Working Papers of the Institute for Empirical Research in Economics can be downloaded in PDF-format from http://www.unizh.ch/iew/wp/

Institute for Empirical Research in Economics, Blümlisalpstr. 10, 8006 Zürich, Switzerland

Phone: 004116343705 Fax: 004116344907 E-mail: bibiewzh@iew.unizh.ch 